\title{
The disc instability model for X-ray transients: Evidence for truncation and irradiation
}

\author{
G. Dubus ${ }^{1, \star}$, J.-M. Hameury ${ }^{2}$, and J.-P. Lasota ${ }^{3}$ \\ 1 Astronomical Institute "Anton Pannekoek", University of Amsterdam, Kruislaan 403, 1098 SJ, Amsterdam, \\ The Netherlands \\ 2 Observatoire de Strasbourg, UMR 7550 du CNRS, 11 rue de l'Université, 67000 Strasbourg, France \\ 3 Institut d'Astrophysique de Paris, 98bis boulevard Arago, 75014 Paris, France
}

Received 10 November 2000 / Accepted 9 February 2001

\begin{abstract}
We study the prospect of explaining the outbursts of Soft X-ray Transients (SXTs) by the thermalviscous instability in a thin disc. This instability is linked to hydrogen ionization and is significantly changed when irradiation of the disc by X-rays from the inner regions is included. We present the first numerically reliable, physically consistent calculations of the outburst cycles which include the effects of accretion disc irradiation. The decay from outburst is governed by irradiation, as pointed out by King \& Ritter (1998), leading to slow exponential decays. At the end of the outburst, the disc is severely depleted, which lengthens the time needed to rebuild mass to the critical density for an outburst. Despite this, the long recurrence times and quiescent X-ray luminosities of SXTs still require the inner disc to be replaced by another type of flow in quiescence, presumably a hot advection dominated accretion flow (ADAF). We include the effects of such truncation and show that the resulting lightcurves are conclusively similar to those of SXTs like A0620-00. We conclude that the two- $\alpha$ disc instability model provides an adequate description of the outbursts of SXTs when both truncation and irradiation are included. The values for the viscosities in outburst and in quiescence are comparable to those used in CVs. We discuss the model in the context of present observations.
\end{abstract}

Key words. accretion - accretion discs - instabilities - stars: binaries: close - X-rays: general

\section{Introduction}

Low mass X-ray binaries (LMXBs) are systems in which a low-mass donor star loses matter to a neutron star or a black hole via Roche lobe overflow. The infalling gas forms an accretion disc where it diffuses towards the central body by losing its angular momentum through viscous interactions. Soft X-ray Transients (SXTs), a sub-class of LMXBs, show large amplitude X-ray and optical outbursts during which their luminosity increases by several orders of magnitude within a few days. The peak is sometimes followed by an exponential decay on a timescale of a month before the system returns to its very low luminosity quiescent state. This "fast-rise exponential-decay" (FRED) behaviour is considered to be typical of SXT lightcurves and is at the focus of our modeling effort. One should keep in mind, however, that many if not most of the SXT outbursts do not have this shape. The recurrence time between outbursts is from several months to tens of years

\footnotetext{
Send offprint requests to: G. Dubus,

e-mail: gd@tapir.caltech.edu

* Present address: Theoretical Astrophysics, Caltech 130-33, Pasadena, CA 91125, USA.
}

(see the reviews of Tanaka \& Shibazaki 1996; Chen et al. 1997). All of the known black hole LMXBs are transient systems.

LMXBs and cataclysmic variables (CVs) show many similar characteristics and the analogy has led to the interpretation of the outbursts of SXTs as being due to a thermal-viscous instability in a geometrically thin, optically thick Keplerian accretion disc, just as in dwarf novae (e.g. Cannizzo et al. 1982). In this disc instability model (DIM), a steady-state flow is stable if hydrogen is everywhere ionized. This would be the case for persistent LMXBs and their CV cousins, the novae-like systems. But if the temperature or (equivalently) the mass accretion rate from the donor star $\dot{M}_{\text {tr }}$ is low enough for hydrogen to recombine, the disc becomes thermally and viscously unstable, oscillating between a hot, ionized state (outburst) and a cold, neutral state (quiescence). This is due to the strong dependence of the opacity on temperature when hydrogen is partially ionized (for reviews of the DIM, see Osaki 1996; Cannizzo 1998a; Lasota 2001).

Unfortunately, in this standard form, the DIM fails to provide an adequate model of LMXBs. For instance, the DIM predicts almost all LMXBs to be transient systems, 
in clear contradiction with observations. Van Paradijs (1996) noted that the X-ray flux coming from (or close to) the compact object could heat the outer accretion disc above the hydrogen ionization temperature and therefore stabilize the flow. There is strong observational evidence of such irradiation e.g. the optical emission of LMXBs which comes mainly from X-rays reprocessed in the outer disc (van Paradijs \& McClintock 1994). Yet, the simple model consisting of a point source irradiating a thin disc does not lead to any significant heating of the outer disc (Dubus et al. 1999; see also Meyer \& Meyer-Hofmeister 1982). The geometry is certainly more complex and may involve indirect rather than direct irradiation of the disc. A corona reflecting only a small fraction $(\sim 0.5 \%)$ of the $\mathrm{X}$-ray luminosity from the center towards the outer disc would result in enough heating to quench the instability. Similarly, a disc warped out of the orbital plane could intercept enough of the central X-ray flux.

Despite this gross uncertainty on the geometry of irradiation heating, there is no doubt that it plays a major role in the physics of LMXBs. The obvious next step in finding a model for SXTs, which we address here, is to examine the consequences of including irradiation heating. This is particularly interesting since the standard DIM completely fails to explain the outbursts of SXTs. Numerical models show outbursts lasting at most only a few tens of days and with short recurrence times (Mineshige \& Wheeler 1989; Menou et al. 2000). Moreover, the standard DIM predicts a rapid decay from the outburst peak instead of a slower exponential decline. This was interpreted as a failure of the standard "two- $\alpha$ " assumption (see Sect. 2.4 below) used to describe the viscous interactions in the DIM (Cannizzo et al. 1995). But King \& Ritter (1998) pointed out that including irradiation heating might naturally lead to the observed exponential decays without resorting to more complex formulations of the viscosity. Previous studies which argued that irradiation should have a negligible influence on the outburst properties (e.g. Cannizzo et al. 1995; Cannizzo 1998b) assumed point source irradiation for which self-shadowing is crucial (Meyer \& Meyer-Hofmeister 1982; Mineshige et al. 1990). We have shown such arguments to be incorrect for persistent LMXBs and, in all likelihood, this also applies to SXTs (Dubus et al. 1999).

Furthermore, it is quite clear that the thin disc cannot extend down to the surface of the compact object in quiescence: the X-ray detection of quiescent SXTs implies mass transfer rates onto the compact object which are four orders of magnitude higher than those predicted by the standard DIM (Lasota 1996). This difficulty is resolved by a two-component accretion flow in which the inner thin disc gradually evaporates into an advectiondominated accretion flow (ADAF) during the decline (Lasota et al. 1996; for reviews, see Lasota 1999; Narayan, Mahadevan \& Quataert 1999; note that a different model was suggested for neutron-star SXTs by Brown et al. 1998). This idea has provided a good framework to understand the spectra of SXTs (e.g. Esin et al. 1997), their quiescent luminosity (e.g. Narayan et al. 1996) and the X-ray/optical delay during the rise of the outburst (Hameury et al. 1997). The evaporated disc does not participate in the thermal-viscous instability and, from a dynamical viewpoint, the disc can essentially be considered as truncated inside.

The outburst lightcurves predicted by the truncated disc instability models (TDIM) have been recently investigated by Menou et al. (2000); (see also Cannizzo 1998b; Meyer \& Meyer-Hofmeister, 1999). Menou et al. showed that, if the quiescent viscosity is smaller (by factors $\sim 4$ ) than the value usually assumed in dwarf novae, the TDIM results are in reasonable agreement with some of the observed properties of SXTs. Standard values of viscosity in quiescence lead to outbursts which are too short, with reflares (see below Sect. 3.1), and to short recurrence times. The major drawback of this model is, however, that the observed disc irradiation is not taken into account.

In this work we show that a modified DIM including the effects of evaporation and irradiation can reproduce reasonably well the outbursts of SXTs with standard values of the viscosity. The assumptions and the numerical technique used in the model are described in Sect. 2. The predictions of the classical DIM are briefly recalled in Sect. 3. We successively explore the effects of irradiation heating without evaporation in Sect. 4 and with evaporation in Sect. 5. In Sect. 6 we complete our investigation by varying different parameters of the full model before discussing the results of this study in Sect. 7 .

\section{Numerical model}

The numerical scheme used is that of Hameury et al. (1998), modified to include irradiation heating as in Dubus et al. (1999) and Hameury et al. (1999). Evaporation of the inner disc is treated in the same way as in Menou et al. (2000).

Basically, the model takes advantage of the fact that in a thin disc one can decouple the vertical structure from the radial evolution equations. The vertical structure equations are solved first, providing a grid from which the heating and cooling terms can be obtained during the actual model computation. The radial disc equations are then solved on an adaptive mesh where the number of grid points is highest in the regions of steep temperature or density gradients. This results in great accuracy and eliminates pure numerical uncertainties which plagued earlier attempts (e.g. Mineshige \& Wheeler 1989; Cannizzo et al. 1995). We discuss here some aspects related to the timedependent irradiated disc model that were not discussed in the above references.

\subsection{Vertical structure}

The series of disc vertical structures, including irradiation, is calculated by solving Eqs. (22-27) of Dubus et al. (1999), assuming thermal balance and given values for the 
radius $R$, the surface column density $\Sigma$, the midplane temperature $T_{\mathrm{c}}$ and the irradiation temperature $T_{\mathrm{irr}}$. In these equations, X-ray heating is limited to a thin layer above the disc and appears in the boundary condition at the surface:

$T_{\mathrm{surf}}^{4}=T_{\mathrm{eff}}^{4}+T_{\mathrm{irr}}^{4}$.

Viscous heating follows the usual $\alpha$ prescription i.e. is proportional to the local pressure in the layer $P$ (Shakura \& Sunyaev 1973):

$\frac{\mathrm{d} F_{\text {vis }}}{\mathrm{d} z}=\frac{3}{2} \alpha \Omega_{\mathrm{K}} P$

where $\Omega_{\mathrm{K}}=\left(G M_{1} / R^{3}\right)^{1 / 2}$ is the Keplerian frequency.

The solutions give for each set of parameters unique values for the effective temperature $T_{\text {eff }}$ and $\alpha$ which are then stored. We compute $70 \times 120 \times 190 \times 9$ vertical structures in $\Omega_{\mathrm{K}}, \Sigma, T_{\mathrm{c}}, T_{\mathrm{irr}}\left(M_{1}\right.$ and $R$ enter only via the Keplerian frequency). We typically consider parameters in the range $10^{8}-10^{12} \mathrm{~cm}$ for $R, 10^{-2}-10^{3} \mathrm{~g} \mathrm{~cm}^{-2}$ for $\Sigma, 10^{3}$ $10^{6} \mathrm{~K}$ for $T_{\mathrm{c}}$, and $0-T_{\mathrm{c}}$ for $T_{\mathrm{irr}}$. In most cases, this samples very well the range of values needed by the disc evolution code.

For given $M_{1}, R$ and $T_{\mathrm{irr}}$, the solutions obtained above show an $S$ shape when plotted in the $\Sigma-T_{\text {c }}$ plane. Examples of such irradiated $S$-curves can be found in Tuchman et al. (1990) and Dubus et al. (1999). The low, cold branch corresponds to a layer of neutral hydrogen while in the upper, hot branch hydrogen is ionized. In between, the gradual ionization of hydrogen is associated with a steep increase of the opacity. This results in the middle branch of the $S$-curve being thermally and viscously unstable. A ring of matter on the unstable branch will evolve either to the hot upper branch or the cold lower branch which are stable. Neighbouring rings are affected somewhat like dominos and the instability propagates, periodically switching the disc between the hot state and cold state. This is the essence of the disc instability model (DIM) whose application to SXTs we study here.

Following are useful numerical fits to the turning points of the $S$-curve: $\Sigma_{\max }, \Sigma_{\min }$ and the associated $T_{\mathrm{c}}$ when irradiation is taken into account. $\Sigma_{\max }$ is the maximum density on the cold, neutral, branch while $\Sigma_{\min }$ is the minimum density of the hot, ionized, branch. We assume that $\alpha$ takes a different value on the hot $\left(\alpha_{\mathrm{h}}\right)$ and on the cold branch ( $\alpha_{\mathrm{c}}$; see below Sect. 2.4).

$$
\begin{aligned}
& \Sigma_{\max }=(10.8-10.3 \xi) \\
& \quad \times \alpha_{\mathrm{c}}^{-0.84} M_{1}^{-0.37+0.1 \xi} R_{10}^{1.11-0.27 \xi} \mathrm{g} \mathrm{cm}^{-2}(3) \\
& T_{\mathrm{c}}\left(\Sigma_{\max }\right)=10700 \alpha_{\mathrm{c}}^{-0.1} R_{10}^{-0.05 \xi} \mathrm{K} \\
& \Sigma_{\min }=(8.3-7.1 \xi) \alpha_{\mathrm{h}}^{-0.77} M_{1}^{-0.37} R_{10}^{1.12-0.23 \xi} \mathrm{g} \mathrm{cm}^{-2}(5)
\end{aligned}
$$

$$
\begin{aligned}
T_{\mathrm{c}}\left(\Sigma_{\min }\right)=(20900 & -11300 \xi) \\
& \times \alpha_{\mathrm{h}}^{-0.22} M_{1}^{-0.01} R_{10}^{0.05-0.12 \xi} \mathrm{K}
\end{aligned}
$$

where $\xi=\left(T_{\mathrm{irr}} / 10^{4} \mathrm{~K}\right)^{2}, M_{1}$ is in solar masses and $R_{10}=R / 10^{10} \mathrm{~cm}$. These fits are very close to those of Hameury et al. (1998) in the case of no irradiation. Equation (29) of Dubus et al. (1999) can be considered as a special case of Eq. (5) above for steady-state and a given dependence of $T_{\text {irr }}$ on $R$. At the $\Sigma_{\text {min }}$ turning point, the maximum of the opacity is reached close to the photosphere while it is at the bottom of the layer at $\Sigma_{\max }$ (Cannizzo \& Wheeler 1984). Irradiation thus has a much stronger influence on $T_{\mathrm{c}}\left(\Sigma_{\min }\right)$ than on $T_{\mathrm{c}}\left(\Sigma_{\max }\right)$.

The $S$-curve disappears when $T_{\mathrm{c}}\left(\Sigma_{\max }\right) \geq T_{\mathrm{c}}\left(\Sigma_{\min }\right)$. Irradiation heating is then enough to fully ionize the layer. This happens for $T_{\mathrm{irr}} \approx 10^{4} \mathrm{~K}$ with a weak dependence on $M_{1}, \alpha$ and $R$ (Tuchman et al. 1990; Dubus et al. 1999). Note that this does not mean the vertical structure becomes isothermal (Dubus et al. 1999). A disc where $T_{\text {irr }}$ is everywhere greater than $10^{4} \mathrm{~K}$ will be thermally and viscously stable.

\subsection{Disc evolution equations}

The disc evolution is governed by the usual vertically integrated radial equations for mass, angular momentum and energy conservation: (Eqs. (1-6) of Hameury et al. 1998; see also Cannizzo 1993) to which we add an equation for $T_{\text {irr }}$ (Eq. (8) below, Sect. 2.3). In particular, the energy conservation equation is:

$\frac{\partial T_{\mathrm{c}}}{\partial t}=\frac{2\left(Q^{+}-Q^{-}+J\right)}{C_{P} \Sigma}-\frac{\Re T_{\mathrm{c}}}{\mu C_{P}} \frac{1}{r} \frac{\partial\left(r v_{\mathrm{r}}\right)}{\partial r}-v_{\mathrm{r}} \frac{\partial T_{\mathrm{c}}}{\partial r}$,

where $Q^{+}$and $Q^{-}$are the surface heating and cooling rates respectively. These are determined using the grid of vertical structures calculated above. The details of the procedure may be found in Sect. 3 of Hameury et al. (1998).

\subsection{Irradiation temperature}

We have shown in Dubus et al. (1999) that the simple assumption of a central irradiating point source in the plane of a thin disc led to results which were incompatible with observations. Following previous work (e.g. Shakura \& Sunyaev 1973) we write the irradiation flux as:

$\sigma T_{\text {irr }}^{4}=\mathcal{C} \frac{L_{x}}{4 \pi R^{2}} \quad$ with $L_{x}=\epsilon \min \left(\dot{M}_{\text {in }}, \dot{M}_{\mathrm{Edd}}\right) c^{2}$

$\dot{M}_{\text {in }}$ is the mass accretion rate at the inner disc radius and $\dot{M}_{\text {Edd }}$ is the Eddington mass accretion rate for an accretion efficiency $\epsilon$ of $0.1: \dot{M}_{\mathrm{Edd}}=1.4 \times 10^{18} M_{1} \mathrm{~g} \mathrm{~s}^{-1}$. The maximum luminosity that can irradiate the disc is thus Eddington-limited. In the models including evaporation (see Sect. 5) $\epsilon$ varies to reflect the very low efficiency of the ADAF. Without evaporation, $\epsilon$ is constant, equal to 0.1 .

$\mathcal{C}$ is a measure of the fraction of the X-ray luminosity that heats up the disc and, as such, contains information on the irradiation geometry, X-ray albedo, X-ray 
spectrum, etc. Kim et al. (1999) used an analogous prescription for their indirect irradiation flux. We found that the observed optical magnitudes and stability properties of persistent low mass X-ray binaries were compatible with a value of $\mathcal{C} \approx 5 \times 10^{-3}$ (with $\epsilon=0.1$, Dubus et al. 1999). Esin and co-workers found some evidence for changes of $\mathcal{C}$ in the outbursts of GRO J1655-40 (a.k.a. V1033 Sco), A0620-00 (a.k.a. V616 Mon) and GRS 1124-68 (a.k.a. GU Mus) (Esin et al. 2000a, 2000b). Radiation-driven warping of the outer disc in long period SXTs would produce a varying value of $\mathcal{C}$ (e.g. Ogilvie \& Dubus 2001). For simplicity, we have nonetheless restricted ourselves to $\mathcal{C}$ constant in this study.

\subsection{Temperature dependence for $\alpha$}

As in our previous works, we assume that $\alpha$ takes on two different values on the hot and cold branches of the $S$ curve. Using a single value for $\alpha$ produces only low amplitude outbursts as numerous previous works have shown.

Since irradiation modifies the hot branch, we adopt a slightly different prescription for the dependence of $\alpha$ on temperature than that used by Hameury et al. (1998, 1999):

$$
\begin{aligned}
\log (\alpha)=\log \left(\alpha_{\mathrm{c}}\right)+ & {\left[\log \left(\alpha_{\mathrm{h}}\right)-\log \left(\alpha_{\mathrm{c}}\right)\right] } \\
\times & {\left[1+\left(\frac{T_{\text {crit }}}{T_{\mathrm{c}}}\right)^{8}\right]^{-1} }
\end{aligned}
$$

where $T_{\text {crit }}=0.5\left[T_{\mathrm{c}}\left(\Sigma_{\max }\right)+T_{\mathrm{c}}\left(\Sigma_{\min }\right)\right]$ is calculated using Eqs. (4) and (6). This ensures that, even with irradiation, $\alpha=\alpha_{\mathrm{h}}$ on the hot branch and $\alpha=\alpha_{\mathrm{c}}$ on the cold branch.

\subsection{Boundary conditions}

The boundary conditions are those of Hameury et al. (1998). We stress that in all models, the outer disc radius $R_{\text {out }}$ is variable. The removal of angular momentum from the disc by the tidal forces from the secondary (as expressed in Hameury et al. 1998) sets $R_{\text {out }}$. The mass transfer rate $\dot{M}_{\text {tr }}$ from the secondary to the accretion disc appears in the outer boundary conditions.

The inner boundary conditions change when evaporation is included and this is discussed below in Sect. 4. In models without evaporation, the inner disc radius is held fixed at a given $R_{\text {in }}$. In our calculations we chose larger values than appropriate if the disc were to extend down to the compact object e.g. to $R_{\text {in }}=3 R_{\mathrm{S}}$ for a black hole primary. This is essentially a numerical limitation due to the very low densities at low radii when the disc enters quiescence (see Fig. 6). This is not a drawback: as stressed in the introduction, there are strong arguments in favour of a truncated disc in quiescence rather than a disc extending down to the compact object.

In outburst $R_{\text {in }}$ could be expected to be close to the compact object but our assumption does not change any of the results. In outburst the inner disc is hot and the viscous timescale at which density perturbations evolve is very short at low radii. Adjustments of the inner disc are therefore instantaneous compared to the evolution timescale as long as $R_{\text {in }}$ is low enough. We have verified this by computing some models with $R_{\text {in }}=10^{7}-10^{9} \mathrm{~cm}$ which showed identical outburst lightcurves. A large $R_{\text {in }}$ also avoids the Lightman-Eardley instability which arises when the radiation pressure dominates in the inner disc. Note however that strong irradiation can suppress this instability (Czerny et al. 1986; Mineshige \& Kusunose 1993).

\subsection{Numerical considerations}

The adaptive mesh numerically resolves the heat fronts and this is one of the major advantages of this scheme (Hameury et al. 1998). The disc mass is not necessarily conserved in the method and therefore provides a test of the numerical accuracy. At least 1200 mesh points were used in the runs shown here, resulting in excellent mass conservation for all cases. The only previous attempt at including irradiation heating in the DIM used at most 21 radial zones, casting very serious doubts on the validity of the results (Mineshige et al. 1990; Kim et al. 1999). Hameury et al. (1998) have emphasized the importance of having numerically resolved heat fronts (i.e. requiring much more than 21 grid points) to obtain reliable results. In another study, Cannizzo (1998) calculated the expected irradiation temperature throughout the eruption cycle of an unirradiated disc but not its feedback on the evolution.

As starting points to our calculations we used the (unstable) steady-state solutions. We then let the disc relax to the periodic outburst-quiescence cycle which is independent of the initial starting point (see Sect. 4.7 of Menou et al. 1999a).

\section{Non-irradiated discs}

The evolution of the inner mass accretion rate times the efficiency gives a measure of the bolometric lightcurve of the system $\left(L_{\mathrm{x}} \approx \epsilon \dot{M}_{\mathrm{in}} c^{2}\right.$ ) (one should in principle convolve this with the detector spectral response to compare with the measured X-ray light curve). In the following, we usually show only the evolution of $\dot{M}_{\text {in }}$, so these are not strictly speaking lightcurves, even though they have the same general characteristics. We show some typical "lightcurves" predicted by the standard DIM for parameters appropriate to soft X-ray transients before turning to models including irradiation.

\subsection{Reflares}

A characteristic feature of the DIM applied to SXTs are the multiple reflares that occur during the outburst decay (Fig. 1). These were previously discussed by Menou et al. (2000). Reflares appear when the surface density $\Sigma$ behind the cooling front is high enough to reach $\Sigma_{\max }$. At the radius at which this happens, the disc becomes thermally unstable and a new heating front develops. This 

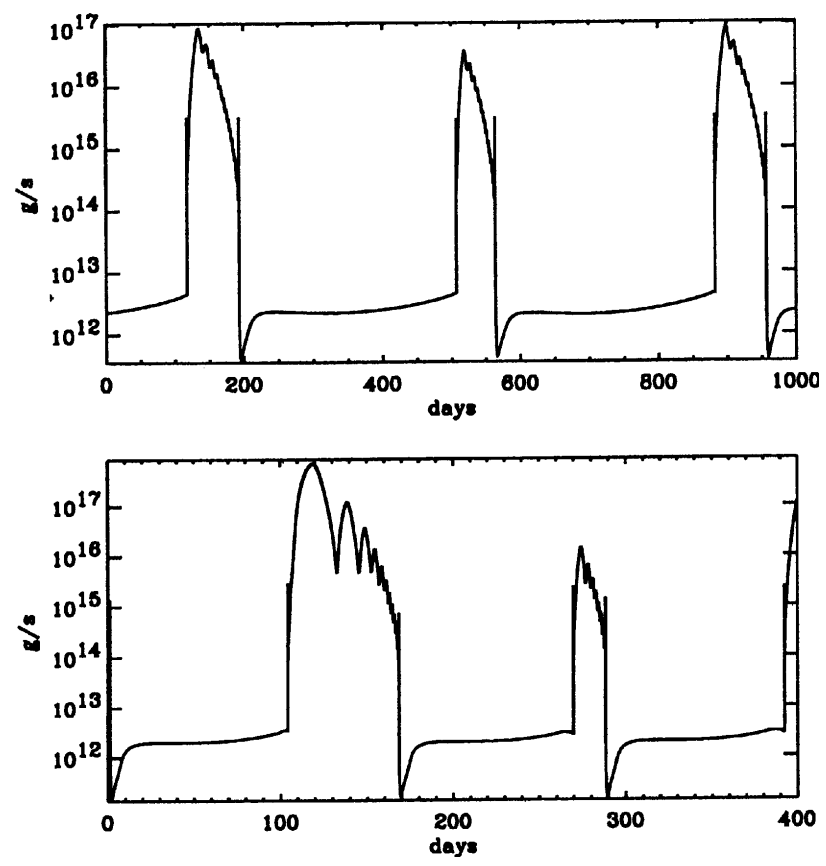

Fig. 1. Typical SXT variations of the inner accretion rate predicted by the standard DIM. This shows the mass accretion at the inner edge of the disc around a $6 M_{\odot}$ black hole (top) and a $1.4 M_{\odot}$ neutron star (bottom). The disc is not irradiated and the inner radius is kept fixed at $R_{\text {in }}=5 \times 10^{8} \mathrm{~cm}$ (top) and $2.5 \times 10^{8} \mathrm{~cm}$ (bottom). The other parameters are those of the "A0620-00" black hole and "Aql X-1" neutron star models of Menou et al. (2000). The standard DIM applied to SXTs is characterized by short recurrence times, low accreted mass during outburst and the presence of strong reflares during the decline. Clearly, this is does not reproduce observations.

front propagates outwards like an inside-out outburst, reheating the disc until $\Sigma(R) \lesssim \Sigma_{\text {min }}$, when cooling can resume. The density in the cold region is depleted as matter is accreted during this process, and the following reflare occurs at smaller radii and have lower amplitudes (Fig. 2).

Menou et al. (1999a) found the density behind the cooling front is roughly $K \Sigma_{\min }$ where $K$ is a function of the viscosity $\alpha$ and of the primary mass $M_{1}$ (Vishniac $1997)$. For $M_{1}=7 M_{\odot}, K \approx 6-7$ so that $K \Sigma_{\min }$ can become greater than $\Sigma_{\max }$ and trigger a heating front. $K$ is lower for lower primary masses $\left(K \approx 4\right.$ for $\left.M_{1}=1.2 M_{\odot}\right)$ making it less likely to have reflares. Menou et al. noted reflares were absent in their calculations with a neutron star primary.

Reflares also appear for neutron stars, but at smaller radii than considered by Menou et al. The reason is that the post-front $\Sigma$ is not strictly proportional to $\Sigma_{\min }$ and has a slightly shallower dependence on radius: $\Sigma \propto R^{0.9}$ for a NS (Fig. 2) instead of $R^{1.1}$ for $\Sigma_{\min }$ and $\Sigma_{\max }$ (Eqs. (3)-(5) without irradiation). Hence there is always a radius for which $\Sigma$ behind the cooling front will become greater than $\Sigma_{\max }$ and ignite a reflare. For a neutron star, the first reflare appears after the cooling front has propagated well inside the disc (Menou et al. 2000, stopped their calculations before this radius was reached) and has a strong effect on the lightcurve as it heats back a significant fraction of the disc (bottom panel of Fig. 1).

\subsection{A deficiency of the DIM}

Reflares are a generic feature of the DIM but do not correspond to observed features in the decay lightcurves of SXTs (Chen et al. 1997). Specifically, the rise-times are much too long in the calculated reflares. The decreasing amplitudes and timescales in-between outbursts are different from the regular mini-outbursts displayed by GRO J0422+32 (a.k.a V518 Per). The number of reflares predicted is also incompatible with the glitches of e.g. A0620-00.

Reflares are therefore a deficiency of the DIM (Menou et al. 2000). Models of dwarf novae are not affected by this problem because the radii of the white dwarf primary $\left(\sim 10^{9} \mathrm{~cm}\right)$ and of the discs (few times $\sim 10^{10} \mathrm{~cm}$ ) do not span a range large enough for reflares to appear. The reflares can be avoided in SXTs if, for instance, the thin disc does not extend much further in than the radius at which reflares ignite. However, a thin disc truncated at too high a radius will be cold and stable rather than transient.

Menou et al. (2000) were able to avoid reflares by using a combination of evaporation (reducing their number) and low quiescent viscosity (increasing the density at which a heating front is ignited $\Sigma_{\max }$ ). Although the recurrence timescale predicted between outbursts was reasonable, the outburst shape did not compare well with FRED-type observed lightcurves, the rise time being too long. In addition, the value for the quiescent viscosity was lower than usually assumed $\left(\alpha_{\mathrm{c}} \sim 5 \times 10^{-3}\right)$. This is not a problem per se considering the present knowledge on viscosity in quiescence (see e.g. Gammie \& Menou 1998) but it could indicate different transport mechanisms in U Gem type dwarf novae with $\alpha_{\mathrm{c}} \sim 0.02$ and SXTs with $\alpha_{\mathrm{c}} \sim 0.005$. However, the mechanisms might be the same, but the efficiencies different. Very low values of $\alpha_{\mathrm{c}}$ have been advocated in the dwarf nova WZ Sge (Smak 1993) which is in many respects similar to SXTs; in this case, the very low value of $\alpha_{\mathrm{c}}$ must be due to a different mechanism, since it is very unlikely that the efficiency of the same mechanism could vary by 2 or 3 orders of magnitude. The following section will show how irradiation by lowering $\Sigma$ behind the cooling front solves this problem without the need for very low values of $\alpha_{\mathrm{c}}$.

\section{Irradiated discs}

In this section we study the effect of including selfirradiation to the standard DIM. We present only one model for this purpose (other may be found in Dubus 2000) with $M_{1}=7 M_{\odot}, \dot{M}_{\mathrm{tr}}=10^{16} \mathrm{~g} \mathrm{~s}^{-1}, \alpha_{\mathrm{h}}=0.2$, $\alpha_{\mathrm{c}}=0.02,<R_{\mathrm{out}}>=10^{11} \mathrm{~cm}$ and a fixed inner radius at $R_{\text {in }}=10^{9} \mathrm{~cm}$ (this is further discussed below in Sect. 4.6). Irradiation is included using Eq. (8) with $\epsilon=0.1$ when $\dot{M}_{\text {in }}>10^{16} \mathrm{~g} \mathrm{~s}^{-1}$ and $\epsilon=0.1\left(\dot{M}_{\text {in }} / 10^{16} \mathrm{~g} \mathrm{~s}^{-1}\right)^{6}$ below. This amounts to a quick cutoff of irradiation below 


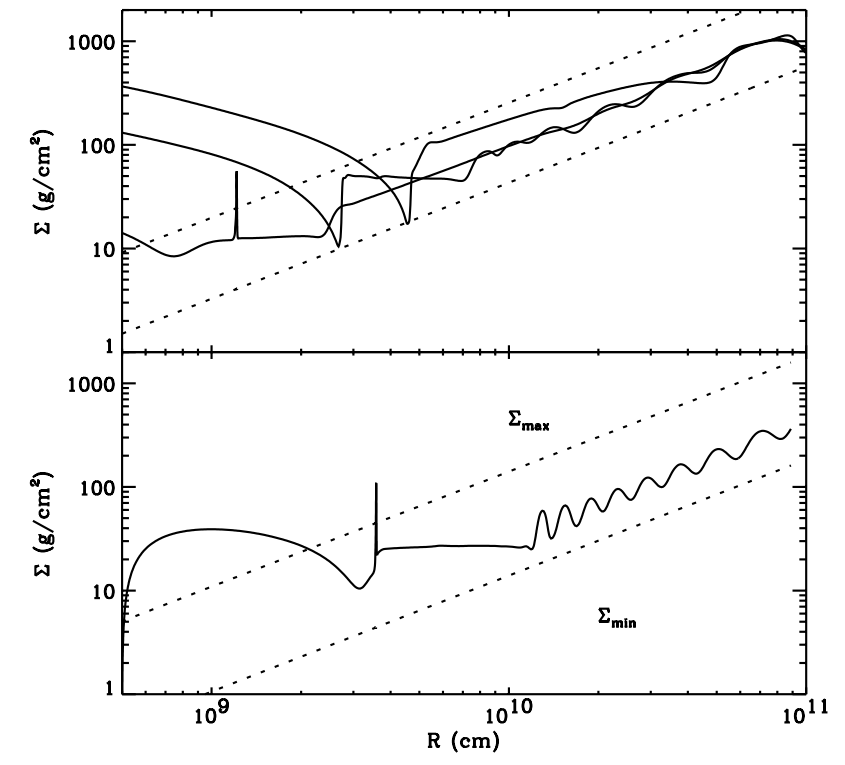

Fig. 2. Reflares in non-irradiated discs around a NS (top panel) and a BH (bottom panel). The full lines are the surface density $\Sigma$ at different times during the decay from outburst (Fig. 1) and the dotted lines are $\Sigma_{\max }$ and $\Sigma_{\min }$ (Eqs. (3), (5)). In the top panel, the situation before the onset of the first reflare is shown by the top curve: $\Sigma$ just behind the cooling front is close to the critical $\Sigma_{\max }$ for which the disc switches back to the hot state. The middle and bottom lines show the profile after several reflares: $\Sigma$ in the outer disc has been depleted by the successive passages of the heat front (which can be seen in the bottom curve). The lower panel shows the situation after many successive reflares in a disc around a BH primary. The wiggles in the density profile corresponding to previous reflares have not yet been smoothed out by viscous diffusion. The inner edge is at $10^{8} \mathrm{~cm}$ (top) and $5 \times 10^{8} \mathrm{~cm}$ (bottom).

$10^{16} \mathrm{~g} \mathrm{~s}^{-1}$. The exact form of $\epsilon$ matters little as long as it ensures that irradiation is only important during the outburst (a reasonable assumption). The resulting outburst time profile is shown in Fig. 3.

\subsection{Rise}

We start with a cold, quiescent disc in which most of the matter has been accreted in a preceding outburst. During quiescence (see below), mass transfer from the secondary replenishes the flow until the density becomes high enough and an annulus arrives at the thermally unstable branch of the $S$-curve. The ring cannot maintain thermal equilibrium and undergoes a transition to the hot branch.

As in the standard DIM, two heating fronts are formed which propagate inwards and outwards from the ignition radius (Menou et al. 1999a and references therein). In "inside-out" (type B) outbursts the ignition radius is small and the propagation time of the inward bound front is very small compared to the other one. In "outside-in" (type A) outbursts, the opposite is true. In the standard DIM, "inside-out" fronts can stall rather easily leading to short, low-amplitude outbursts. The density profile in

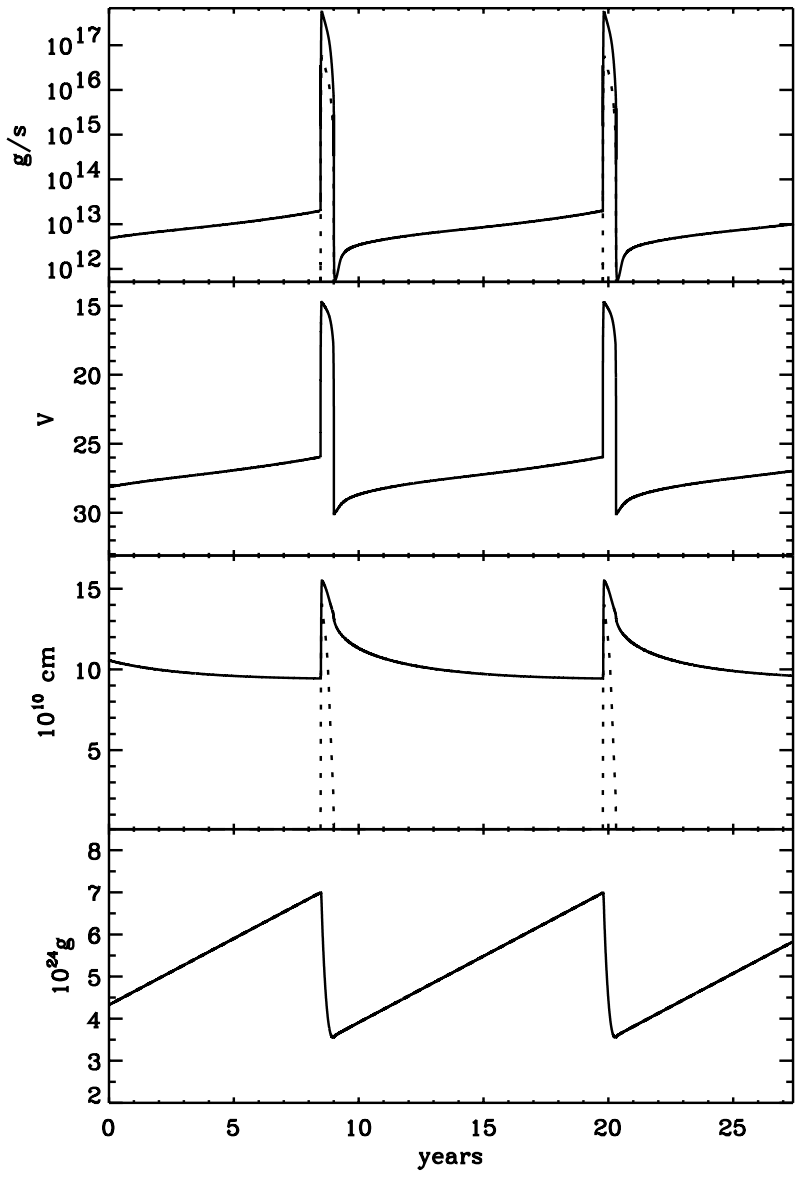

Fig. 3. Example of an outburst cycle when irradiation is included. From top to bottom: the mass accretion rate at the inner edge $\dot{M}_{\text {in }}$ (full line) and $\dot{M}_{\text {irr }}=\epsilon \dot{M}_{\text {in }}$ (dotted line), the $V$ magnitude, the outer disc radius $R_{\text {out }}$ (full line) and the transition radius $R_{\text {trans }}$ between the hot and the cold regions (dotted line), the mass of the disc $M_{\text {disc }}$. The parameters are $M_{1}=7 M_{\odot}, \dot{M}_{\mathrm{tr}}=10^{16} \mathrm{~g} \mathrm{~s}^{-1}, \alpha_{\mathrm{h}}=0.2, \alpha_{\mathrm{c}}=0.02$, $\left.<R_{\text {out }}\right\rangle=10^{11} \mathrm{~cm}$ and a fixed inner radius at $R_{\text {in }}=10^{9} \mathrm{~cm}$. Details of the outbursts and the evolution of the density and temperature profiles can be seen in Figs. 5-7.

quiescence is generally not far from $\Sigma_{\max } \propto R$ so that, as the outward front progresses through the disc, it encounters regions of higher densities and $\Sigma_{\max }$ (the critical density needed to raise a ring to the hot branch). If the front does not transport enough matter to raise the density at some radius above $\Sigma_{\max }$, it stalls and a cooling front develops. "Outside-in" fronts always progress through regions of decreasing $\Sigma_{\max }$ and therefore always heat the whole disc. Another consequence is that inside-out fronts propagate slowly, leading to slow rise times. This is particularly evident in Fig. 8 of Menou et al. (2000).

Irradiation does not change the structure of the heating front (compare Fig. 4 with Fig. 1 of Menou et al. 1999a) but does change the maximum radius to which an inside-out outburst can propagate. Since we do not consider self-screening, the outer disc always sees the irradiation flux from the central regions. As an inside-out front propagates, $\dot{M}_{\text {in }}$ rises and the outer cold disc is 
increasingly irradiated (see Fig. 5). Irradiation heating reduces the critical density needed to reach the hot branch (Eq. (3)), easing front propagation. Obviously, a larger hot region implies a greater optical flux and irradiation always lowers the peak optical magnitude.

In an inside-out outburst, the outer regions the front has not yet reached are frozen on the timescale on which $\dot{M}_{\text {in }}$ evolves (see Fig. 5). If $\dot{M}_{\text {in }}$ (hence $\dot{M}_{\text {irr }}$ ) increases on timescales shorter than the thermal timescale in the cold disc, this can lead to situations where $T_{\text {irr }}>T_{\mathrm{c}}$ at some radii. The vertical heat flux changes sign and would require negative values of $\alpha$ in our treatment of thermal imbalance i.e. the assumptions of the code break down. We assumed that such an annulus is dominated by irradiation i.e. is isothermal at $T_{\mathrm{c}}$ and that irradiation contributes an additional heating term to the radial thermal equation (Eq. (7)) $Q_{\text {add }}^{+}=\sigma\left(T_{\text {irr }}^{4}-T_{\text {c }}^{4}\right)$ to reflect the imbalance at the photosphere between the outgoing flux $\sigma T_{\mathrm{c}}^{4}$ and the incoming flux $\sigma T_{\mathrm{irr}}^{4}$. This, or other assumptions, actually had very little influence on the rise-to-outburst lightcurve. Further studies of this phenomenon would require a detailed model of irradiation in SXTs where the geometry, the exact flux and spectrum of irradiating photons are properly set out. Note also that the absence of any back loop in the equations to prevent this situation may suggest that some additional physics is needed. One possibility is that rapidly increasing irradiation would evaporate the upper layers of the disc (Begelman et al. 1983; Hoshi 1984; Idan \& Shaviv 1996; de Kool \& Wickramasinghe 1999).

Figure 5 shows the evolution of the $\Sigma$ and $T_{\mathrm{c}}$ radial profiles during the outburst rise. As the front reaches the outer edge of the disc, the profiles in the hot region converge to those of a steady disc with constant $\dot{M}(R)$. This is because the viscous timescale, which is inversely proportional to $\alpha T_{\mathrm{c}}$, becomes short enough to equilibrate the mass flow in the hot region (e.g. Menou et al. 1999a, and references therein). Irradiation has little influence on the actual vertical structure in this region as discussed in Dubus et al. (1999) and we find $T_{\mathrm{c}} \propto \Sigma \propto R^{-3 / 4}$ as in a non-irradiated steady disc. Only in the outermost disc regions does the vertical structure become irradiationdominated, i.e. isothermal.

The peak accretion rate (and optical magnitudes) is rather difficult to estimate analytically since it depends on the maximum radius to which the heat front can propagate. Qualitatively, this is set mostly by the conditions in the disc at the onset of the outburst. The principle factor is the total disc mass when the outburst starts $M_{\max }$ which is constrained by $\Sigma_{\max }$. Parameter studies (Sect. 6) show that any changes which result in a lower $\Sigma_{\max }$, hence in a lower $M_{\text {disc }}$ do lead to smaller outburst peaks. Increasing $M_{1}, \alpha_{\mathrm{c}}{ }^{1}$ or decreasing $R_{\text {out }}$ give smaller $\dot{M}_{\text {peak }}$. Increasing

${ }^{1}$ The dependence is really on the ratio $\alpha_{\mathrm{h}} / \alpha_{\mathrm{c}}$. The mass in the disc is almost the same between the beginning and the peak of the outburst. Assuming $M_{\max } \propto \Sigma_{\max } \propto \alpha_{\mathrm{c}}^{-0.8}$
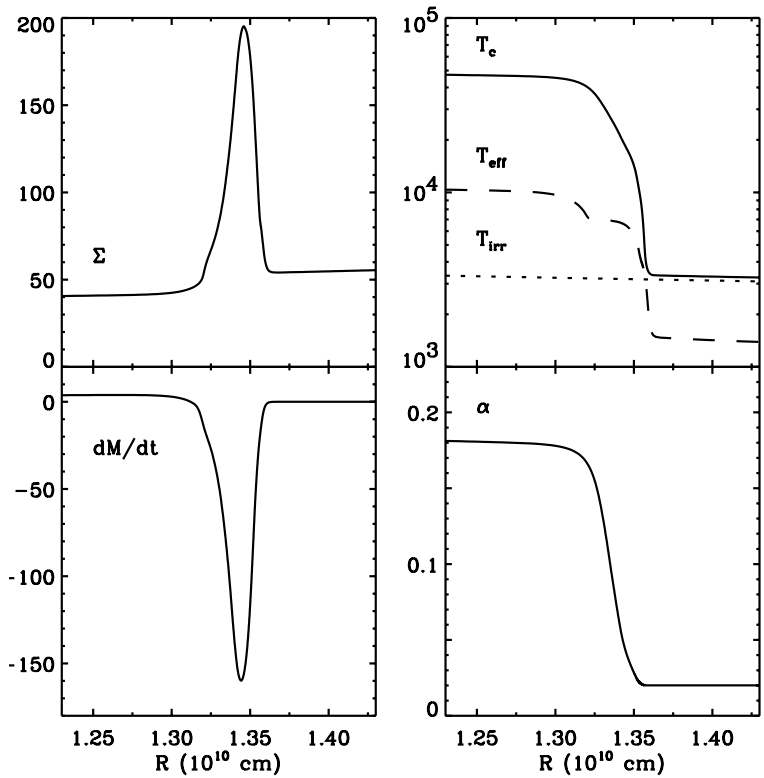

Fig. 4. An "inside-out" heating front in an irradiated disc. This is the front at $t \approx 1.5$ days in Fig. $5 . \Sigma$ is in $\mathrm{g} \mathrm{cm}^{-2}$, temperatures are in $\mathrm{K}, \dot{M}$ is in units of $10^{16} \mathrm{~g} \mathrm{~s}^{-1}$. The cold outer disc is almost isothermal with $T_{\mathrm{c}} \approx T_{\mathrm{irr}}$.

the mass transfer rate leads to higher disc masses in quiescence and higher peaks.

In any case, $\dot{M}_{\text {peak }}$ should be lower than or of order of the critical mass accretion rate $\dot{M}_{\text {crit }}$ corresponding to $\Sigma_{\min }$ at $R_{\text {out }}$, as the disc is close to steady state at maximum if it has been brought entirely to a hot state. If the heating front has not been able to reach the outer edge, $\dot{M}_{\text {peak }}$ will be lower.

\subsection{Decay from outburst maximum}

The outburst decay can generally be divided into three parts:

- First, outer disc X-ray irradiation inhibits the coolingfront propagation. Since more mass is lost than gained from the secondary $\left(\dot{M}_{\text {in }} \gg \dot{M}_{\text {tr }}\right)$ the disc is drained by viscous accretion of matter (King \& Ritter 1998);

- Second, the accretion rate becomes low enough that the X-ray irradiation is unable to prevent the cooling from propagating. The propagation speed of this front is, however, controlled by irradiation;

- Irradiation plays no role and the cooling front switches off the outburst on a local thermal time.

We now discuss these three phases in more detail.

\subsubsection{Viscous "exponential" decay}

In the model presented here, the disc becomes fully ionized at the outburst peak. The disc then evolves with $\dot{M}(R)$

$\overline{\text { and } M_{\max } \propto \dot{M}_{\mathrm{peak}}^{0.7} \alpha_{\mathrm{h}}^{-0.8}}$ (Shakura-Sunyaev steady disc) shows $\dot{M}_{\text {peak }} \propto\left(\alpha_{\mathrm{h}} / \alpha_{\mathrm{c}}\right)^{8 / 7}$. 
almost constant so that $\nu \Sigma \sim \dot{M}_{\text {in }}(t) / 3 \pi$; the total mass in the disc is thus

$M_{\mathrm{d}}=\int 2 \pi R \Sigma \mathrm{d} R \propto \dot{M}_{\mathrm{in}} \int \frac{2}{3} \frac{R}{\nu} \mathrm{d} R$

and one can consider that the disc decays through a sequence of quasi-stationary states. In irradiation dominated discs as well as in standard Shakura-Sunyaev discs, $\nu \propto T \propto \dot{M}^{\beta /(1+\beta)}$, with $\beta=3 / 7$ in hot Shakura-Sunyaev discs, and $\beta=1 / 3$ in irradiation dominated discs. The outer disc radius does not vary much so the time evolution of the disc mass is:

$$
\frac{\mathrm{d} M_{\mathrm{d}}}{\mathrm{d} t}=-\dot{M}_{\mathrm{in}} \propto M_{\mathrm{d}}^{1+\beta}
$$

showing that $\dot{M}_{\text {in }}$ evolves almost exponentially, as long as $\dot{M}_{\text {in }}^{\beta}$ can be considered as constant (i.e. over about a decade in $\dot{M}_{\text {in }}$, as found by King \& Ritter 1998 for irradiated discs, and by Mineshige et al. 1993 for nonirradiated discs with angular momentum removal). Powerlaws with indices close to -1 are found in discs with constant angular momentum, i.e. in which there are no tidal torques preventing the outer disc radius to expand indefinitely (Lyubarskii \& Shakura 1987; Cannizzo et al. 1990; Mineshige et al. 1993), and are of no practical interest here.

The decay is viscous as long as thermal equilibrium can be maintained. Incidentally, this makes it difficult, if not impossible, to have a viscous decay in the standard DIM without additional assumptions. In outside-in outbursts, matter accumulates at the outer disc edge and the resulting density excess decreases viscously, producing "flat-top" light curves. Thermal equilibrium requires the whole disc to be kept in the hot state. If the outer disc is cold then there will be a thermally unstable region evolving rapidly (i.e. non-viscously, see next paragraph). Keeping a disc hot can be achieved by additional mass transfer during the outburst or non-standard sources of heating (e.g. tidal heating as in Buat-Ménard et al. 2001). A smaller $\alpha_{\mathrm{c}}$ increases the difference between $\Sigma_{\max }$ and $\Sigma_{\min }$ and hence can also help keep the disc in the hot state longer (Menou et al. 2000).

Irradiation heating not only leads to the exponential decay but also provides a natural way to keep the disc hot. An irradiation temperature at the outer edge above $10^{4} \mathrm{~K}$ ensures hydrogen is everywhere ionized. For such temperatures, the disc is thermally stable whatever the local density $\Sigma$ and accretion rate. Put differently, the lower branch of the $S$-curve disappears when $T_{\text {irr }} \gtrsim 10^{4} \mathrm{~K}$ (see Figs. $4-5$ of Dubus et al. 1999) or $\Sigma_{\min }=\Sigma_{\max }$ at $T_{\text {irr }} \approx 10^{4} \mathrm{~K}$ (and are undefined below: see Eqs. (3), (5)).

One should remember, however, that many SXT lightcurves are not exponential, despite their discs being irradiated. Clearly additional physical processes have to be taken into account if one wishes to explain such "nontypical" behaviour (see e.g. Esin et al. 2000a).

\subsection{Irradiation-controlled linear decay}

The second stage of the decay starts when a disc ring cannot maintain thermal equilibrium and switches to the cool lower branch of the $S$-curve. The disc outer edge always becomes unstable first with $\Sigma<\Sigma_{\min }$ since $\Sigma \propto R^{-3 / 4}$ (see above) while $\Sigma_{\min } \propto R$. In an irradiated disc this happens when the central object does not produce enough X-ray flux to keep the $T_{\text {irr }}\left(R_{\text {out }}\right)$ above $10^{4} \mathrm{~K}$ so that the low state is again possible. The material in the low state has a lower viscosity and piles up, leading to the appearance of a cooling front with a width $w \propto H$ (Papaloizou \& Pringle 1985; Meyer 1986; Fig. 4 of Menou et al. 1999a). The front propagates through the disc at a speed $V_{\text {front }} \approx \alpha_{\mathrm{h}} c_{\mathrm{s}}$ (Meyer 1984; Vishniac \& Wheeler 1996). In the standard DIM, the sound speed depends on the temperature at the transition between the hot and cold regions and is almost constant as verified by numerical calculations (Menou et al. 1999a).

In an irradiated disc, however, the transition between the hot and cold regions is set by $T_{\text {irr }}$ since the cold branch only exists for $T_{\text {irr }} \lesssim 10^{4} \mathrm{~K}$. Unlike a non-irradiated disc, the cooling front can propagate only as far as the radius at which $T_{\mathrm{irr}} \approx 10^{4} \mathrm{~K}$, i.e. as far as there is a cold branch to fall onto. Here also irradiation controls the decay. The hot region stays close to steady-state but with a shrinking size $R_{\text {hot }} \sim \dot{M}_{\text {in }}^{1 / 2}$ (Eq. (8) with $T_{\text {irr }}\left(R_{\text {hot }}\right)=$ const.). The mass redistribution during front propagation is complex with the hot region losing mass both through inflow and outflow and our models show steeper declines than what some analytic approximations predict (e.g. King 1998).

The linear decay is illustrated in Fig. 6. The irradiation temperature is lower than $10^{4} \mathrm{~K}$ at the outer edge for $t>70$ days where the decay becomes steeper. The cooling front (see also Fig. 8) appears as a depression propagating inwards in the surface density profile. The irradiation temperature and the critical densities $\Sigma_{\min \text {,max }}$ are plotted for the last profile at $t \approx 190$ days, showing the cooling front is at the radius for which $T_{\mathrm{irr}} \approx 10^{4} \mathrm{~K}$ and that $\Sigma_{\min \text { max }}$ are undefined for lower radii.

\subsection{Final thermal decay}

The quick decay after $t>190$ days is due to vanishing irradiation as $\epsilon$ becomes very small for $\dot{M}_{\text {in }}<10^{16} \mathrm{~g} \mathrm{~s}^{-1}$. The cooling front thereafter propagates freely inwards, on a thermal time scale.

\subsection{Mini-reflares}

As discussed above, the edge of the hot zone is at the stability limit set by $T_{\text {irr }} \approx 10^{4} \mathrm{~K}$. At this point, one has $\Sigma \approx \Sigma_{\min } \approx \Sigma_{\max }$ because irradiation modifies the critical surface densities. Further away from the edge of the hot zone, the irradiation temperature decreases rapidly $\left(T_{\mathrm{irr}}^{4} \propto\right.$ $R^{-2}$ ) and $\Sigma_{\max }$ gradually becomes greater than $\Sigma_{\min }$. In the non-irradiated limit, the ratio $\Sigma_{\max } / \Sigma_{\min }$ is a constant depending only on the ratio $\alpha_{\mathrm{c}} / \alpha_{\mathrm{h}}$ (see e.g. Fig. 2). 

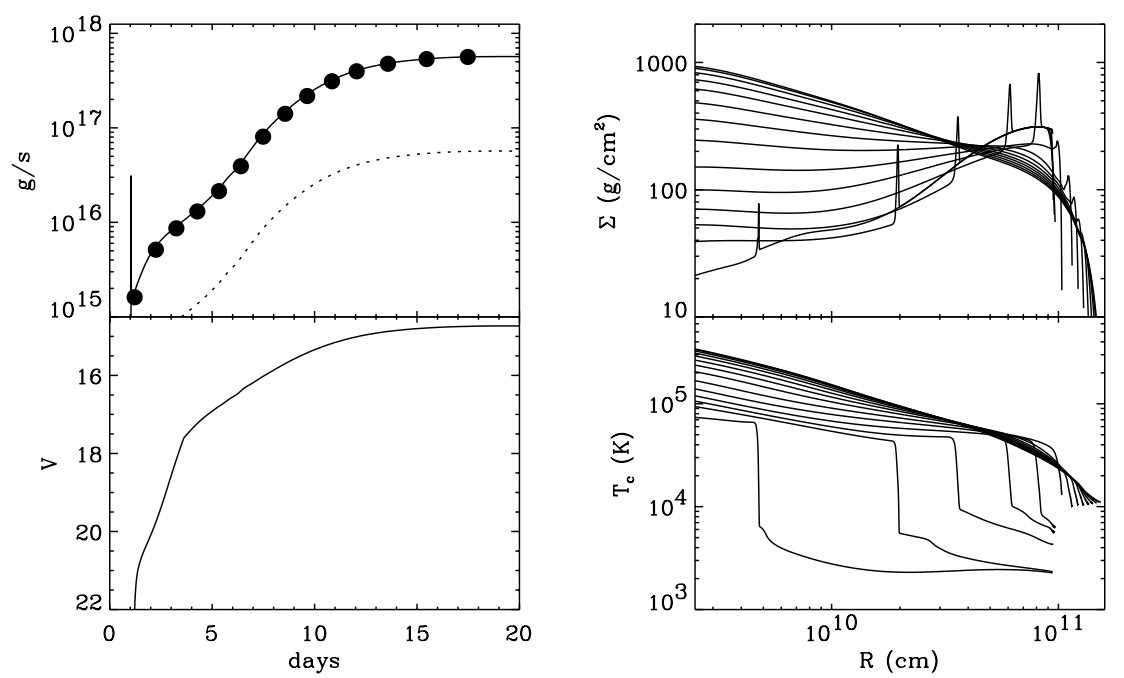

Fig. 5. The outburst rise for the model of Sect. 4 (irradiated, with a fixed $\left.R_{\text {in }}\right)$. The upper left panel shows $\dot{M}_{\text {in }}$ and $\dot{M}_{\text {irr }}$ (dotted line); the bottom left panel shows the $V$ magnitude. The spike at $t \approx 1$ day corresponds to the arrival at $R_{\text {in }}$ of the inward propagating front. The rise is dominated by the outward front (inside-out outburst). Each dot corresponds to one of the $\Sigma$ and $T_{\mathrm{c}}$ profiles in the right panels. The disc expands during the outburst to transport the angular momentum of the material being accreted. The profiles close to the peak are those of a steady-state $\operatorname{disc}\left(\Sigma \propto T_{\mathrm{c}} \propto R^{-3 / 4}\right)$.
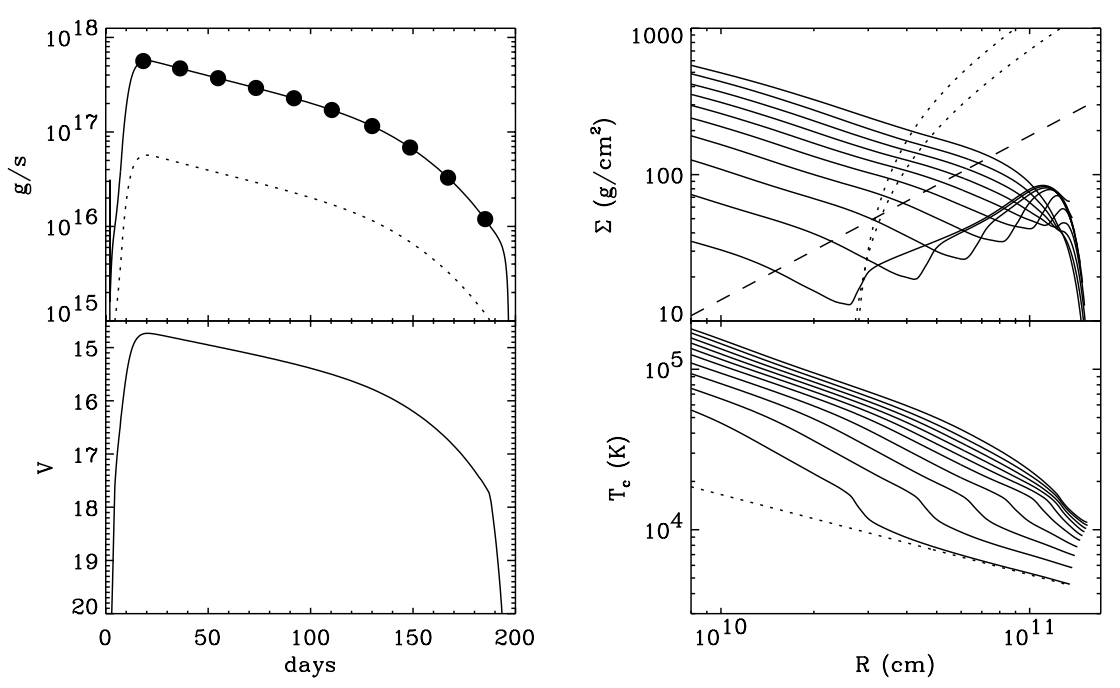

Fig. 6. The outburst decay for the model discussed in Sects. 4.2.1-4.3. The fully ionized disc decays viscously (exponential lightcurve) until $t \approx 70 \mathrm{~d}$ where $T_{\text {irr }}\left(R_{\text {out }}\right) \approx 10^{4} \mathrm{~K}$. Thereafter the outer disc can fall on the cold branch of the $S$-curve and a cooling front appears, propagating inward only as far as $T_{\mathrm{irr}} \approx 10^{4} \mathrm{~K}$ (irradiationcontrolled linear decay). At $t \approx 190 \mathrm{~d}$ irradiation shuts off and the disc cools quickly. The dashed line is $\Sigma_{\min }$ for $\alpha_{\mathrm{h}}$ without irradiation, showing the postfront $\Sigma$ are very low when irradiation is included. $\Sigma_{\min , \max }\left(\alpha, T_{\mathrm{irr}}\right)$, and $T_{\text {irr }}$ are shown for the last $\Sigma$ and $T_{\mathrm{c}}$ profiles (dotted lines).
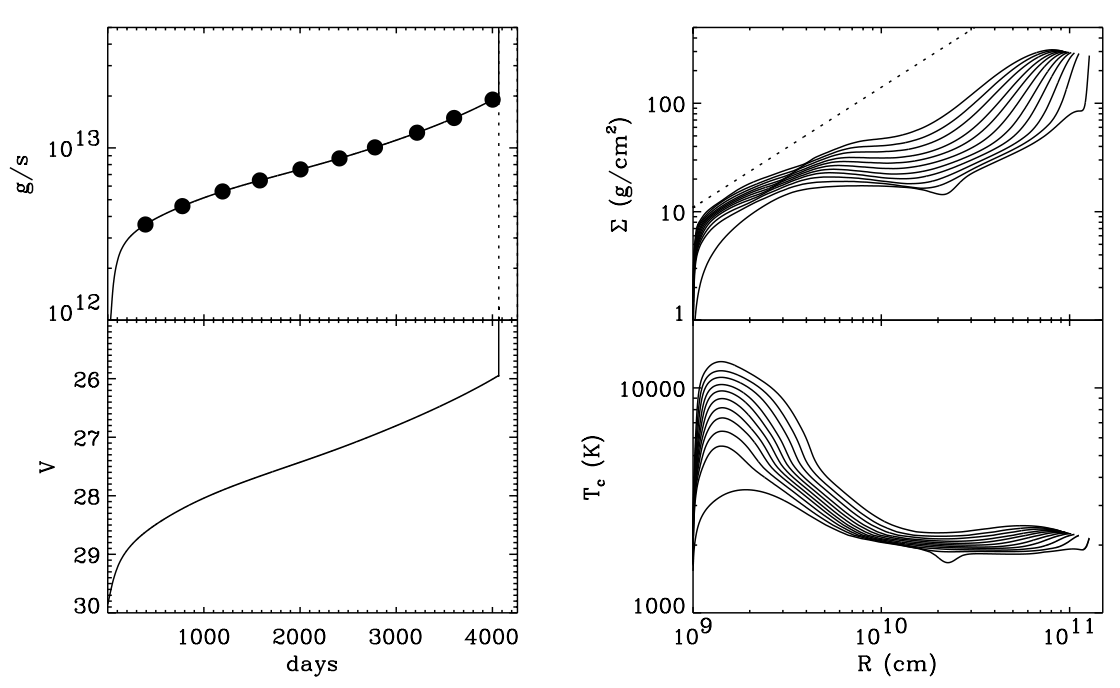

Fig. 7. Quiescence for the model discussed in Sect. 4.6. The irradiation flux is negligible in quiescence and the overall evolution is the same as in the standard DIM. Mass transfer from the secondary is slow enough that matter diffuses down the disc, gradually increasing $\dot{M}_{\text {in }}$. The outburst is triggered inside at $R \approx 2 \times 10^{9} \mathrm{~cm}$ when $\Sigma$ reaches $\Sigma_{\max }$ (dotted line). Lower densities at the beginning of the quiescent state (due to the irradiation-controlled outburst decay) lead to a long recurrence time (about 10 years).

The region immediately behind the cooling front where $\Sigma_{\max }$ is close to $\Sigma_{\min }$ is clearly very unstable. Slight variations of $\Sigma$ may suffice to have $\Sigma>\Sigma_{\max }$ behind the cooling front and therefore start a reflare just as in a nonirradiated disc. This depends on the numerical details of the model, such as the functional of $\alpha$ with temperature (Sect. 2.4).

These mini-reflares are unimportant. The critical density $\Sigma_{\min }$ increases quickly behind the cooling front (see Fig. 8) because the irradiation flux decreases with $R^{-2}$. 

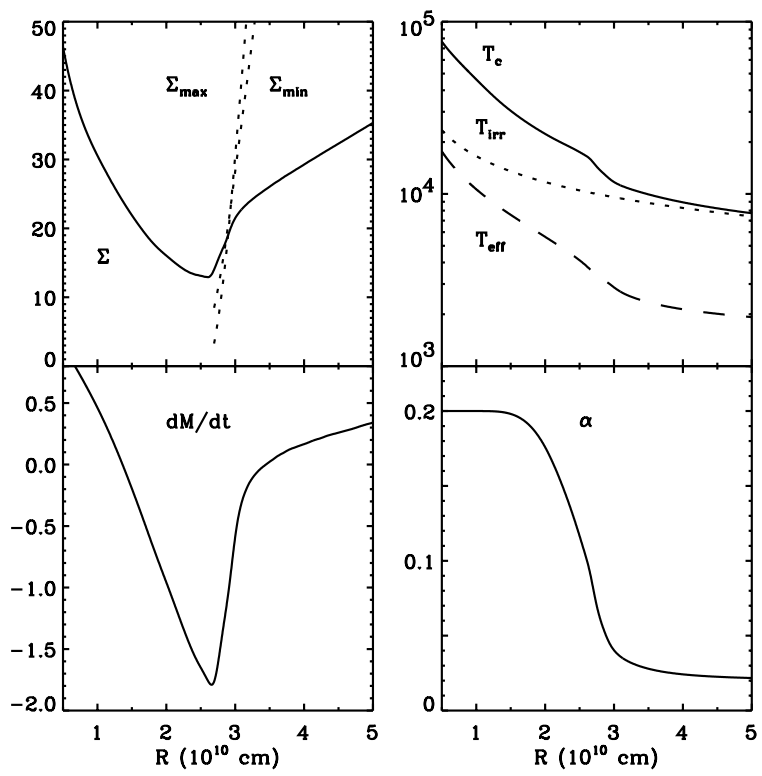

Fig. 8. The cooling front in an irradiated disc. This is the front at $t \approx 190$ days in Fig. 6. $\Sigma$ is in $\mathrm{g} \mathrm{cm}^{-2}$, temperatures are in $\mathrm{K}, \dot{M}$ is in units of $10^{16} \mathrm{~g} \mathrm{~s}^{-1}$. The front is at the position for which $T_{\text {irr }}$ (dotted line) is $\approx 10^{4} \mathrm{~K}$. At this point $\Sigma_{\min } \approx \Sigma_{\max }$ since there is no cold branch for higher $T_{\mathrm{irr}}$ (smaller $R$ ). As $T_{\mathrm{irr}}$ decreases the two critical $\Sigma$ separate and converge to their nonirradiated values (this can be seen in Fig. 6; note the values of $\Sigma_{\max }$ and $\Sigma_{\min }$ shown here are only accurate to the precision of the fits given by Eq. (3), (5)). The cold outer disc is almost isothermal with $T_{\mathrm{c}} \approx T_{\mathrm{irr}}$. The negative values of $\dot{M}$ show the cooling front transports matter to the outer disc.

Therefore, the heating front in a mini-reflare reaches almost immediately a radius where $\Sigma<\Sigma_{\min }$ and cooling resumes. Furthermore, irradiation seriously depletes the disc and the front finds very little matter to fuel its propagation. $\Sigma_{\min }$ is much lower than its non-irradiated value (Fig. 6). For instance, Fig. 4 of Dubus et al. (1999) shows $\Sigma_{\min }\left(T_{\mathrm{irr}} \approx 10^{4} \mathrm{~K}\right) \approx 30 \mathrm{~g} \mathrm{~cm}^{-2}$ instead of $\Sigma_{\min }\left(T_{\mathrm{irr}}=\right.$ $0 \mathrm{~K}) \approx 150 \mathrm{~g} \mathrm{~cm}^{-2}$. The much lower post-cooling front densities prevent large reflares as in the standard DIM. In practice, mini-reflares in irradiated discs have no influence on the lightcurve (very low amplitudes and cycles, see Fig. 9) but can be a numerical nuisance.

\subsection{Quiescence}

As discussed above, irradiation dramatically slows down the cooling process. The decay lasts much longer and, accordingly, the total mass accreted during an outburst is much larger. The densities after propagation of the cooling front are low so the total mass of the disc as it enters quiescence is much less than in the non-irradiated case.

The low quiescent surface densities imply low midplane temperatures. The $\left(\Sigma, T_{\mathrm{c}}\right) S$-curves show that for low $\Sigma$ and no irradiation (as appropriate once the disc enters quiescence) the lower branch is flat with $T_{\mathrm{c}} \approx 2000 \mathrm{~K}$ whatever $R$ or $M_{1}$ (see e.g. in Figs. 4, 5 of Dubus et al. 1999). In contrast, the standard DIM leads to higher $\Sigma$,

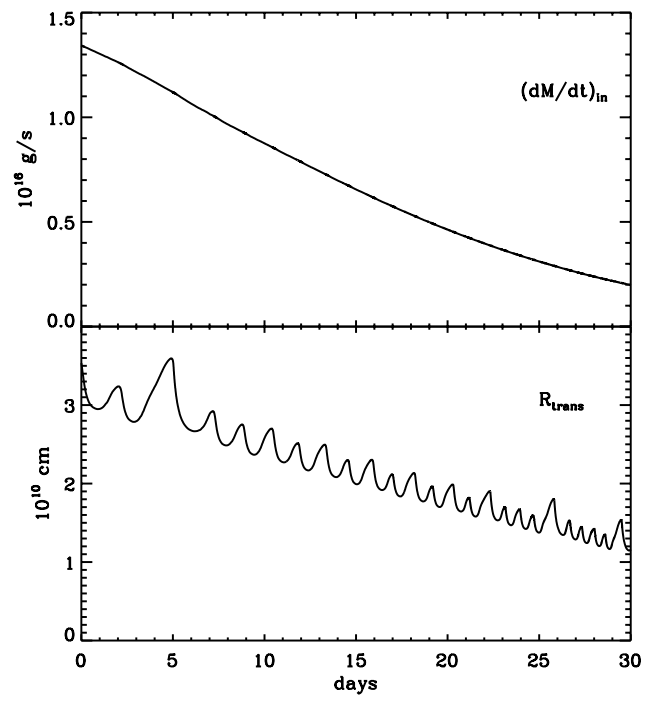

Fig. 9. Example of mini-reflares in an irradiated disc. The region close to $T_{\mathrm{irr}} \approx 10^{4} \mathrm{~K}$ is highly unstable with $\Sigma_{\min } \approx \Sigma_{\max }$ (Fig. 8). This can lead to a succession of heat fronts propagating quickly back and forth in a small region (bottom panel). The exact details depend on the model assumptions (e.g. $\alpha$ ). This has no influence on either $\dot{M}_{\text {in }}$ (top panel) or the optical flux (not shown). See Sect. 4.5 for details.

close to the non-irradiated $\Sigma_{\min }$ and thus to $T_{\mathrm{c}} \approx 4000 \mathrm{~K}$ (e.g. Osaki 1996; Menou et al. 2000).

The critical density $\Sigma_{\max }$ that the disc must reach before an outburst can start will be the same as in the standard DIM if irradiation in quiescence is negligible. This is in general true since $\dot{M}_{\text {in }} \propto T_{\text {irr }}^{4}$ is very small ${ }^{2}$. Since the quiescent disc after an irradiation-controlled decay is always less massive, the time to build up the critical density will be longer.

In the model presented in this section, $T_{\text {irr }}=0$ in quiescence. The evolution of the $\Sigma$ and $T_{\mathrm{c}}$ profiles (Fig. 7) shows that matter arriving from the secondary at the outer edge diffuses down the cold disc. The disc contracts due to addition of this lower angular-momentum material. The temperature is almost constant in most of the disc with $T_{\mathrm{c}} \approx 2000 \mathrm{~K}$. This is not true in the later stages of quiescence where $T_{\mathrm{c}}$ rises in the inner disc due to the build up of matter there: the inner rings move up along the lower branch of the $\left(\Sigma, T_{\mathrm{c}}\right) S$-curve and out of the flat $T_{\mathrm{c}} \approx 2000 \mathrm{~K}$ region discussed above. In the last profile, $\Sigma$ is close to $\Sigma_{\max }$ (dotted line) and the outburst is ignited at $R \approx 2 \times 10^{9} \mathrm{~cm}$.

The ignition radius depends essentially on the mass transfer rate from the secondary and the disc's size (Smak 1984). For low $\dot{M}_{\mathrm{tr}}$, matter drifts down the cold disc and $\Sigma_{\max }$ is reached in the inner disc (inside-out, type B outburst). As $\dot{M}_{\mathrm{tr}}$ increases, the mass accumulation time at the outer radius can become lower than the drift time, triggering an outburst in the outer disc (an outside-in,

${ }^{2}$ The quiescent disc can be irradiation-dominated if $\mathcal{C}$ is very large (see Sect. 6.1). In this case, the critical $\Sigma_{\max }$ is lowered by irradiation and the recurrence timescale is shortened. 
type A outburst). The accumulation time is (Ichikawa \& Osaki 1994; see also Osaki 1995, 1996 and Lasota 2001):

$t_{\mathrm{acc}}=\frac{2 \pi R_{\mathrm{out}} \Sigma_{\max }\left(R_{\mathrm{out}}\right)}{\dot{M}_{\mathrm{tr}}} \Delta R$

A ring of material spreads viscously over $\Delta R \approx\left(\nu t_{\text {acc }}\right)^{1 / 2}$ during $t_{\mathrm{acc}}$. Setting $t_{\mathrm{acc}}=t_{\text {drift }}$ gives an estimate of the accretion rate for which the transition from type $\mathrm{B}$ to $\mathrm{A}$ outbursts is expected. Using Eq. (3) and $t_{\mathrm{drift}}=\delta t_{\mathrm{vis}}(\delta$ is a numerical correction factor introduced by Osaki 1996) outside-in outbursts are therefore expected when

$$
\begin{aligned}
\dot{M}_{\text {tr }} \gtrsim 2 \pi R_{\text {out }} \delta^{-1 / 2} \nu \Sigma_{\text {max }}\left(R_{\text {out }}\right) \\
\gtrsim 3.3 \times 10^{16}\left(\frac{\alpha}{0.02}\right)^{0.2}\left(\frac{R_{\text {out }}}{10^{11} \mathrm{~cm}}\right)^{2.6} \\
\quad \times\left(\frac{M_{1}}{7 M_{\odot}}\right)^{-0.9}\left(\frac{T_{\mathrm{c}}}{2000 \mathrm{~K}}\right) \mathrm{g} \mathrm{s}^{-1}
\end{aligned}
$$

with $\delta=1$. This is smaller than the mass accretion rate needed to stabilize an irradiated disc with these parameters $\left(\dot{M}_{\mathrm{tr}} \gtrsim 10^{17} \mathrm{~g} \mathrm{~s}^{-1}\right.$, Eq. (30) of Dubus et al. 1999). In principle type A outbursts are thus possible in SXTs, but numerical calculations show that real outside-in outbursts starting far out in a large disc are difficult to obtain. Osaki (1996) finds $\delta \approx 0.05$ provides an adequate fit to his calculations. The quiescence time of our model (about 4000 days) implies $t_{\text {drift }} \approx 0.1 t_{\text {vis }}\left(R_{\text {out }}\right)$ so that the required $\dot{M}_{\text {tr }}$ for a type A outburst is above the accretion rate for which the disc is stable.

The inner edge of the accretion disc plays a crucial role in quiescence. For low accretion rates $\Sigma_{\max }$ is reached in the inner disc. Since $\Sigma_{\max } \propto R$ the amount of mass needed to trigger an outburst is reduced when the disc inner radius is smaller: the higher $R_{\text {in }}$ in quiescence, the longer the recurrence time. Even with $R_{\text {in }}=10^{9} \mathrm{~cm}$ the model is still short of providing

- the long recurrence timescale $\left(t_{\mathrm{rec}} \approx 10\right.$ years compared to the tens of years inferred in SXTs) and

- the quiescent X-ray luminosities: the predicted quiescent accretion rate, in the range $10^{12-13} \mathrm{~g} \mathrm{~s}^{-1}$ is still too small to account for observed X-ray luminosities exceeding $10^{32} \mathrm{erg} \mathrm{s}^{-1}$.

The next section will show that including disc evaporation into a hot, low-density, accretion flow can solve these problems.

\section{Irradiated discs with evaporation}

Following the work of Menou et al. (2000), we now assume that the inner disc is gradually evaporated into a hot, radiatively inefficient accretion flow during the decay from outburst. The exact nature of the flow is not important as long as it does not participate in the dynamics of the thin accretion disc. A good assumption is that the inner disc is replaced by an advection-dominated accretion flow $(\mathrm{ADAF}) . \mathrm{ADAF}+$ thin disc models have been successful in explaining the spectral states of SXTs and in particular, the quiescent X-ray flux provided the mass accretion rate in the ADAF is large enough (e.g. Esin et al. 1997).

The accretion timescale in an ADAF becomes of the order of the thermal timescale so that the inner flow can be considered as quasi-steady and does not participate in the limit cycle. A good assumption in our dynamical study is therefore to treat evaporation as a variation of the inner radius of the thin disc we model. We take the same rate of evaporation as in Menou et al. (2000):

$$
\dot{M}_{\text {ev }}(R)=0.08 \dot{M}_{\text {Edd }}\left[\left(\frac{R}{R_{\mathrm{s}}}\right)^{1 / 4}+\mathcal{E}\left(\frac{R}{800 R_{\mathrm{s}}}\right)^{2}\right]^{-1} .
$$

The inner radius of the disc is defined as:

$\dot{M}_{\text {ev }}\left(R_{\text {in }}\right)=\dot{M}_{\text {in }}$.

Below this radius all the matter evaporates. Because of the steep dependence of $\dot{M}_{\mathrm{ev}}$ on $R$, we can safely neglect evaporation above this radius. As noted in Menou et al. (2000), the detailed functional dependence of $\dot{M}_{\mathrm{ev}}(R)$, or of $R_{\text {in }}\left(\dot{M}_{\text {in }}\right)$ has essentially no effect on the results; what matters is the value of the inner disc radius during quiescence.

Equation (14) is entirely ad hoc, and is not based on any particular physical mechanism. There are models that, in principle, allow for the determination of the evaporation rate as a function of radius. However, the variety of models shows that there is no general agreement on the physical cause of evaporation. For example, Meyer \& Meyer-Hofmeister (1994) assume that electron conduction from the disc to the corona plays a major role, whereas Shaviv et al. (1999) consider a thermal instability related to the opacity law. In addition, even for a given model, the evaporation law depends on parameters which are not easily measured (as for example the magnetic field and its coherence in the case of electron conduction; Meyer et al. 2000). As all these models have been proposed to explain the existence of holes in observed accretion discs, they, by definition, tend to produce similar results, i.e. the truncation radius must be at a detectable distance from the compact objet. And of course, for the same reason, Eq. (14) would also reproduce these results. The latest calculations by Meyer et al. (2000) give evaporation rates of the order of $1.4 \times 10^{16} \mathrm{~g} \mathrm{~s}^{-1}$ at $10^{10} \mathrm{~cm}$ from a $6 M_{\odot}$ black hole, and $1.6 \times 10^{17} \mathrm{~g} \mathrm{~s}^{-1}$ at $10^{9} \mathrm{~cm}$. These are larger by a factor $\sim 15$ and $\sim 2$ respectively than the values we are using here. Note that the evaporation rate is so high in Meyer et al. (2000) that the quiescent disc would be truncated at too large a radius to be unstable.

The ADAF efficiency roughly scales as $\dot{M}$ (i.e. the luminosity scales as $\dot{M}^{2}$, Esin et al. 1997) . From Eq. (14), this is equivalent to $\epsilon \propto R_{\text {in }}^{-2}$. In the model, we assume $\epsilon=0.1$ when $R_{\text {in }}=R_{\min }$ (in outburst) and $\epsilon=0.1 \times\left(R_{\min } / R_{\text {in }}\right)^{-2}$ when $R_{\text {in }} \geq R_{\min }$ (in quiescence). Irradiation is usually negligible in quiescence so these assumptions have little importance there. Varying $\epsilon$ may 
change slightly the end of the outburst when evaporation becomes important.

We took $R_{\min }=5 \times 10^{8} \mathrm{~cm}$ in all the following models. As discussed below in Sect. 5.1, the only time $R_{\min }$ is reached is in outburst where the inner disc is in thermal equilibrium and close to steady-state. The inner disc edge plays no role in this case.

In the following we discuss in some detail the same model as in Sect. 4 but including evaporation. The overall outburst time profile is shown in Fig. 10. A comparison with Fig. 3 shows the outburst is almost identical but that the disc spends much more time in quiescence $\left(t_{\mathrm{rec}} \approx 21\right.$ years $)$. The maximum luminosity reached during outburst is also increased.

\subsection{The outburst}

After an outburst is triggered, the mass accretion rate at the inner edge gradually increases as in the previous model. The inner radius of the thin disc decreases until its minimum value is reached for $\dot{M}_{\mathrm{in}}=\dot{M}_{\mathrm{ev}}\left(R_{\mathrm{min}}\right)$. A slight change of slope is associated with this (at the 4th dot in Fig. 11). The time $t_{\text {rise }}$ the disc needs to reach $R_{\text {min }}$ is a viscous timescale. Before the onset of the outburst, $R_{\text {in }} \approx 6 \times 10^{9} \mathrm{~cm}$. Therefore, $t_{\text {rise }}$ is of the order of the difference between $t_{\text {vis }}$ at $6 \times 10^{9} \mathrm{~cm}$ and $5 \times 10^{8} \mathrm{~cm}$. The viscous timescale is given by

$t_{\mathrm{vis}}=\frac{R^{2}}{\nu}=\left(G M_{1} R\right)^{1 / 2} \frac{\mu m_{\mathrm{H}}}{\alpha k T_{\mathrm{c}}}$.

For parameters appropriate to this case $\left(T_{\mathrm{c}} \approx 10^{5} \mathrm{~K}, \alpha=\right.$ $\left.0.2, \mu=0.5, M_{1}=7 M_{\odot}\right)$ the difference gives about 6 days which is in good agreement with the numerical calculation. Figure 11 shows the minimum radius is reached at $t \approx$ 5.5 days, about 3 days after the onset of the outburst.

The subsequent evolution is identical to the model where the inner radius is kept fixed. The peak accretion rate and optical flux are higher in the model with evaporation because of the different quiescent histories of the discs. When the outburst starts, the discs do not have the same density distribution and total mass (Figs. 3, 10).

The decay from outburst is also identical to the previous model with a viscous and a linear decay (Fig. 12). The linear decay has a limited influence on the lightcurve since the cooling front only propagates to $8 \times 10^{10} \mathrm{~cm}$ before evaporation sets in $(t \approx 160$ days in Fig. 12$)$. Irradiation then shuts off with the decreasing $\epsilon$ and the front cools the whole disc quickly. At the same time, the inner disc radius rises with the increasing evaporation which also quickens the cooling. In general, evaporation and/or changes in the irradiation efficiency $\epsilon$ take place for $\dot{M}_{\text {in }} \sim \dot{M}_{\text {tr }}$ at which point the total disc mass is close to its minimum value (the disc starts filling in again) and there is little matter left to accrete. Therefore, the exact prescriptions for $\dot{M}_{\text {evap }}$ and $\epsilon$ do not have a significant impact on the outburst lightcurve. For instance, these produce a difference of only a few days in the outburst lengths between the model of Sect. 4 and the model of this section.

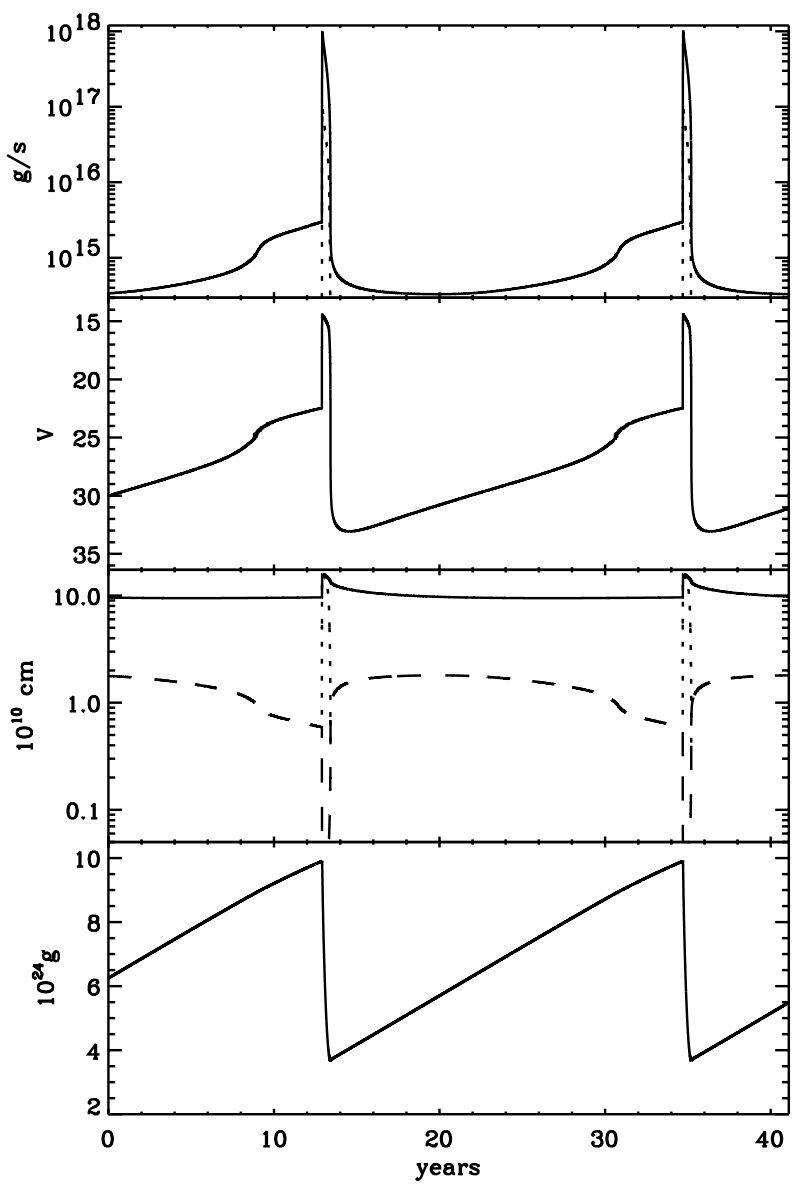

Fig. 10. Example of an outburst cycle when irradiation and evaporation are included. The parameters of the model are the same as in Fig. 3. From top to bottom: $\dot{M}_{\text {in }}$ (full line) and $\dot{M}_{\text {irr }}$ (dotted line); $V$ magnitude; $R_{\text {out }}$ (full line), $R_{\text {trans }}$ (dotted line) and $R_{\text {in }}$ (dashed line), $M_{\text {disc }}$. Details of the outburst and the evolution of the density and temperature profiles can be seen in Figs. 11-13.

\subsection{Quiescence with evaporation}

The whole disc becomes cold and enters quiescence when the cooling front reaches the varying inner radius $R_{\text {in }}$. In Fig. 12 this happens at $t \approx 195$ days when $R_{\text {in }} \approx$ $5 \times 10^{10} \mathrm{~cm}$ and $\dot{M}_{\mathrm{in}} \approx 5 \times 10^{15} \mathrm{~g} \mathrm{~s}^{-1}$. The drift time of cold matter is long and the disc cannot maintain this high accretion rate: $\dot{M}_{\text {in }}$ decreases as material is gradually accreted. This adjustment happens on a long timescale (cold material) which depends on the $\Sigma$ profile left after the outburst. With a fixed $R_{\text {in }}, \dot{M}_{\text {in }}$ is already very low (about $10^{12} \mathrm{~g} \mathrm{~s}^{-1}$, Fig. 7) when the whole disc becomes cold. This is actually a necessary condition for the disc at low radii to reach the cold branch. For such low accretion rates, the supply of material is enough for $\dot{M}_{\text {in }}$ to increase steadily throughout quiescence.

This first stage lasts until material from the outer edge had enough time to drift to the inner edge and increase $\dot{M}_{\text {in }}$. In Fig. 13 this happens at $t \approx 3000$ days. The first three $\Sigma$ radial profiles of Fig. 13 clearly show mass from the outer edge diffusing inwards and reaching 
the inner edge at $t \approx 2500$ days. The disc then steadily builds up mass until the combination of a decreasing $R_{\text {in }}$ (hence $\Sigma_{\max }$ ) and increasing $\Sigma$ triggers an outburst (see also Cannizzo 1998b, Meyer \& Meyer-Hofmeister 1999).

Under most circumstances (Sect. 4.6) the outburst will be of the inside-out type. The reason is that, because of the very large disc sizes of SXTs, the time it takes for matter to diffuse down the disc is shorter than the accumulation time at the outer disc. A truncated disc does not change this conclusion: evaporation does not prevent inside-out type B outbursts.

However, evaporation does suppress the small minioutbursts which are found when the inner disc radius is small (see e.g. Hameury et al. 1998). With $R_{\text {in }}$ fixed at $10^{9} \mathrm{~cm}, \Sigma_{\max }$ is small, of the order of $10 \mathrm{~g} \mathrm{~cm}^{-2}$, and an outburst can be triggered easily as soon as matter diffuses in to the inner radii. This leads to sequences of minioutbursts which are not observed. A truncated disc will need to build up more mass before an outburst can start. The critical density $\Sigma_{\max }$ varies with $R$ and will be much higher when $R_{\text {in }}$ is large, of the order of a $100 \mathrm{~g} \mathrm{~cm}^{-2}$ at $10^{10} \mathrm{~cm}$. This prevents small outbursts from being triggered.

A truncated disc at the onset of an outburst will be more massive than a non-truncated disc (compare Figs. 3 and 10). But since the disc in outburst cools under the same conditions (dictated by $T_{\text {irr }}$ ), the mass of the disc at the end of the outburst will be roughly the same in both cases. If $\Delta M$ is the mass accreted during the outburst then we have $\Delta M_{\text {trunc }}>\Delta M_{\text {no trunc }}$.

The quiescence time is the time it takes to replenish the mass lost during the outburst:

$t_{\text {quiesc }}=\frac{\Delta M}{\dot{M}_{\mathrm{tr}}-\dot{M}_{\mathrm{in}}}$.

Irradiation depletes the disc during the decay, increases $\Delta M$ and leads to longer $t_{\text {quiesc. }}$ In contrast to models in which the disc extends to low radii, truncated discs can have larger $\dot{M}_{\text {in }}$ in quiescence implying longer $t_{\text {quiesc }}$. Equation (17) also shows that the quiescence time depends on the mass transfer rate even for inside-out outbursts when $\dot{M}_{\text {in }}$ in quiescence is a significant fraction of $\dot{M}_{\mathrm{tr}}$. In a disc with low quiescent $\dot{M}_{\text {in }}, t_{\text {quiesc }}$ for inside-out outbursts is of the order of $t_{\text {diff }}$ which is independent of $\dot{M}_{\mathrm{tr}}$ (Osaki 1996; Smak 1993).

\section{Parameter study}

\subsection{Changing the irradiation strength $\mathcal{C}$}

We show in Fig. 14 the effects of a stronger or weaker irradiation on the outbursts. The strength of irradiation is set by the value of $\mathcal{C}$ and we show models for $\mathcal{C}=10^{-3}, 5 \times 10^{-3}, 10^{-2}, 10^{-1}$. As irradiation grows stronger, $\Sigma_{\min }$ becomes lower (see Eq. (5)) and the first effect is to increase the length of the outburst: more mass gets accreted before $\Sigma_{\min }$ is reached. Thus, the relative amount of matter accreted during outburst, $\Delta M / M$, increases with $\mathcal{C}$. The disc spends more time accreting in quiescence before it can build up enough density somewhere to reach $\Sigma_{\max }$.

Like $\Sigma_{\min }, \Sigma_{\max }$ also decreases with increasing irradiation (see Eq. (3)). Therefore, one would also expect $\Sigma_{\max }$ to be reached more easily, hence to have shorter quiescent times. Usually the irradiation flux is low at the end of quiescence so only large values of $\mathcal{C}$ can heat the disc enough to change $\Sigma_{\max }$ (see Fig. 15).

There are two competing effects: (i) the increased amount of matter accreted during an outburst lengthens the quiescence time; (ii) unusually strong irradiation in quiescence lowers the critical density needed to reach an outburst, which reduces the quiescence time. This happens when $\mathcal{C}$ is large, in which case irradiation is no longer negligible in quiescence (in contrast to the models of Sects. 4-5). The numerical models show that there is a $\mathcal{C}_{\max }$ for which the recurrence time is greatest, $\mathcal{C}_{\max } \approx 5 \times 10^{-3}$, i.e. the "standard" value for which persistent low-mass X-ray binaries are stabilized (Dubus et al. 1999). This is probably no more than a coincidence as $\mathcal{C}_{\text {max }}$ certainly changes as a function of $M_{1}, R_{\text {out }}$, etc.

The average mass in the disc $\left\langle M_{\mathrm{d}}\right\rangle$ decreases with stronger irradiation. $\left\langle M_{\mathrm{d}}\right\rangle$ depends mostly on the mass of the disc in quiescence. Since $\Sigma<\Sigma_{\max }$ in quiescence and $\Sigma_{\max }$ decreases with irradiation, $\left\langle M_{\mathrm{d}}\right\rangle$ decreases as well. Another consequence of stronger irradiation are the higher optical fluxes in outburst due to the higher outer disc temperatures.

\subsection{Changing the viscosity $\alpha$}

The effect of changing $\alpha_{\mathrm{h}}$ and $\alpha_{\mathrm{c}}$ is straightforward. The $\alpha$ parameter sets the viscous time of the accretion flow (Eq. (16)). A disc with a low viscosity in quiescence diffuses mass slowly to the inner edge. Therefore, the time to reach $\Sigma_{\max }$ is longer when $\alpha_{\mathrm{c}}$ is lower (see top panel of Fig. 16). This also modifies the peak accretion rate of the outburst. Similarly, a disc with a higher viscosity in outburst accretes mass more quickly and the decay time is shorter (Eqs. (10)-(11)). The conditions for the disc to enter quiescence and the subsequent evolution are independent of $\alpha_{\mathrm{h}}$. Therefore, the models shown in the top panel of Fig. 16 which have different $\alpha_{\mathrm{h}}$ but the same $\alpha_{\mathrm{c}}$ have the same recurrence time.

\subsection{Changing the mass transfer rate $\dot{M}_{\mathrm{tr}}$}

Next, we explore the effect of changing the mass transfer rate $\dot{M}_{\mathrm{tr}}$, keeping all other parameters identical. For inside-out outbursts, the time spent in quiescence with fixed $R_{\text {in }}$ is the diffusion time of matter from the outer to the inner edge, which does not depend on $\dot{M}_{\mathrm{tr}}$. However, models including evaporation show a dependence on $\dot{M}_{\mathrm{tr}}$ when $\dot{M}_{\text {in }}$ in quiescence is not negligible compared to $\dot{M}_{\mathrm{tr}}$ 

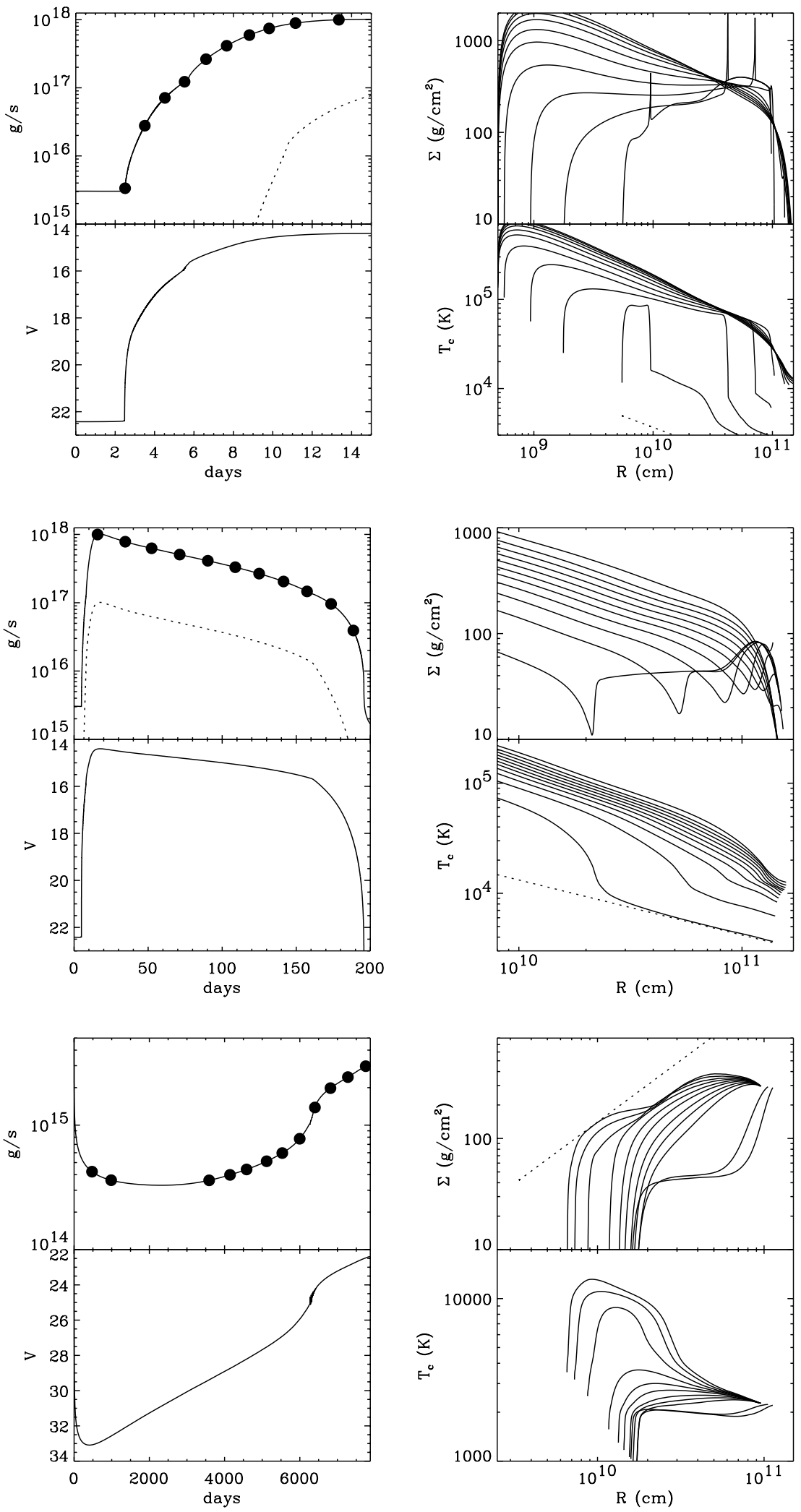

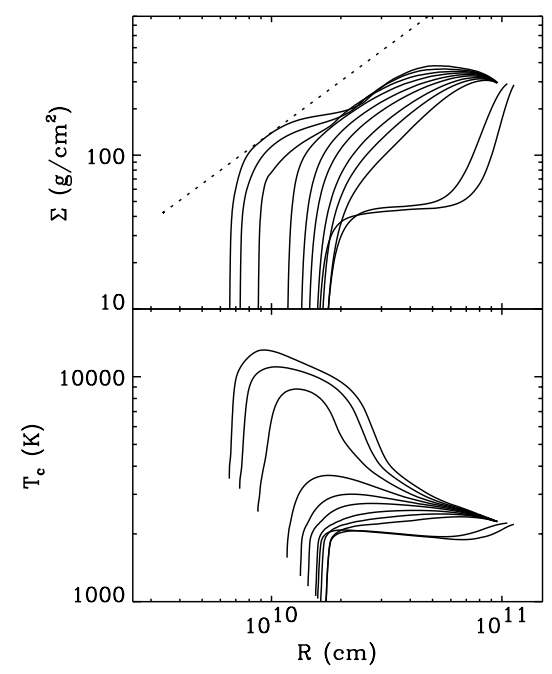

Fig. 11. The outburst rise for the model of Sect. 5 which includes irradiation and evaporation (see Sect. 5.1). Upper left panel shows $\dot{M}_{\text {in }}$ and $\dot{M}_{\text {irr }}$ (dotted line); bottom left panel shows the $V$ magnitude. Right panels show radial profiles at the different times indicated by the black dots on the curves. Evaporation decreases during the first part of the rise with the thin disc extending to smaller radii. At $t \approx 5.5$ days the thin disc reaches the minimum possible inner radius of the model. The disc then behaves in exactly the same way as in Fig. 5 .

Fig. 12. The outburst decay for the model discussed in Sect. 5.1. The disc behaves in the same way as in Fig. 6 until evaporation sets in at $t \approx 170$ days $\left(\dot{M}_{\text {in }}=\dot{M}_{\text {evap }}\left(R_{\text {min }}\right)\right)$. This cuts off irradiation and the disc cools quickly. In contrast to Fig. 6, the irradiation cutoff happens before the cooling front could propagate through most of the disc, hence the irradiation-controlled linear decay ( $t \approx 80-170$ days) is less obvious in the time profile. $T_{\mathrm{irr}}$ (dotted line) is shown for the last temperature profile.

Fig. 13. Quiescence for the model discussed in Sect. 5.2. The irradiation flux is negligible in quiescence. Overall, the evolution is the same as in the standard DIM. Mass transfer from the secondary is slow enough that matter diffuses down the disc, gradually increasing $\dot{M}_{\text {in }}$ (and lowering $R_{\text {in }}$ ). The outburst is triggered at $R \approx 10^{10} \mathrm{~cm}$ when $\Sigma$ reaches $\Sigma_{\max }$ (dotted line). Lower densities at the beginning of the quiescent state (due to the irradiationcontrolled outburst decay) lead to a long recurrence time (about 35 years).
(Eq. (17)). Figure 17 shows the quiescence time shortens with increasing $\dot{M}_{\mathrm{tr}}$.

Higher $\dot{M}_{\text {tr }}$ result in longer outbursts since (i) the companion provides more material during the outburst and (ii) the total disc mass at the onset of the outburst increases with $\dot{M}_{\mathrm{tr}}$. The conditions in the disc are the same at the end of the outburst, regardless of the mass transfer rate (except, of course, at the outer edge) because cooling happens in the same way for discs of the same size. A ring at a given radius can only cool when $T_{\text {irr }}$ reaches $10^{4} \mathrm{~K}$ and the surface density behind the front is roughly the same in all cases. Thus, even with different $\dot{M}_{\mathrm{tr}}$, the discs 

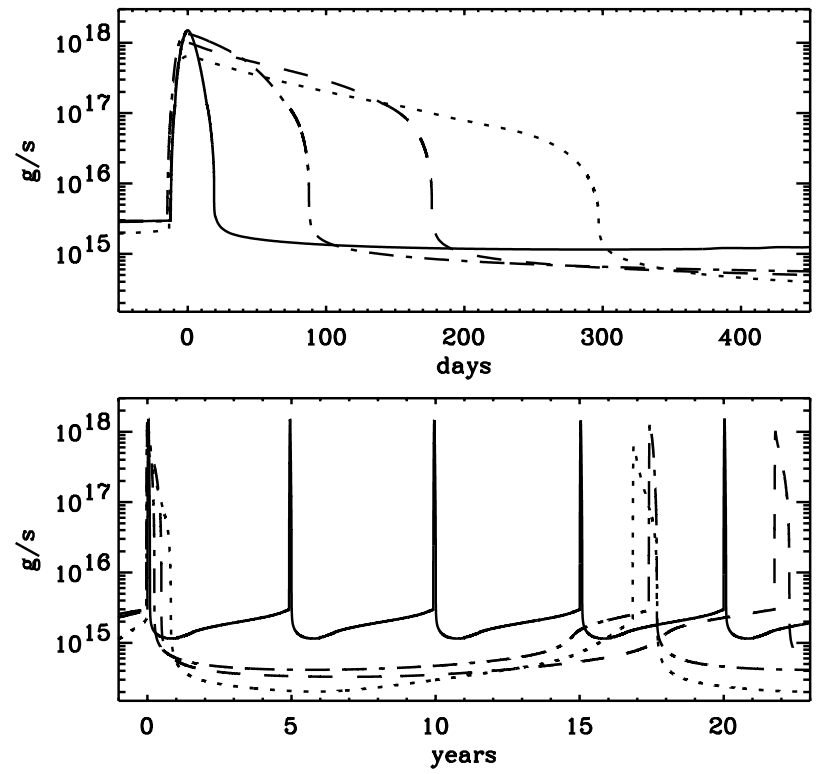

Fig. 14. Effect of changing the irradiation parameter $\mathcal{C}$ on the outburst time evolution and cycle (see Sect. 6.1 for details). $\mathcal{C} / 10^{-3}=0$ (full line), 1 (dash-dotted), 5 (dashed), 100 (dotted). All other parameters are identical to those of the model described in Sect. 4. The non-irradiated curve shows no reflares in this particular case. This is in general not the case, even with evaporation: the same model with $M_{1}=6 M_{\odot}$ shows them.

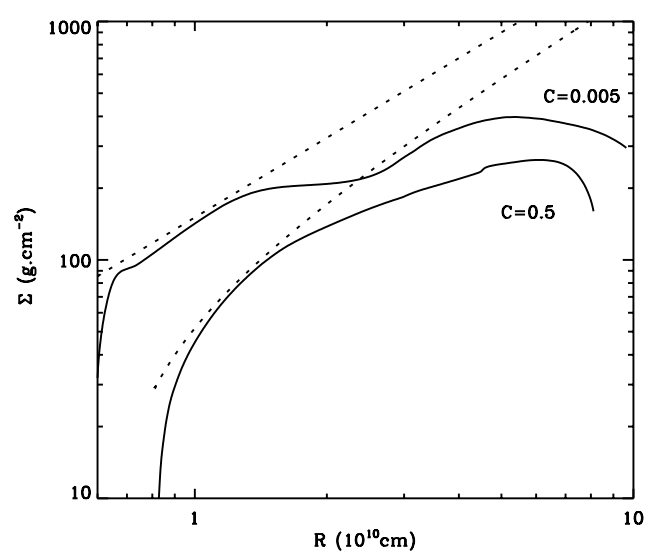

Fig. 15. Density profiles $\Sigma(R)$ in quiescent evaporated discs before the onset of an outburst. The dotted line is $\Sigma_{\max }$. For $\mathcal{C}=5 \times 10^{-3}$ irradiation is negligible in quiescence and the critical $\Sigma_{\max }$ is the same as for a non-irradiated disc. For larger values of $\mathcal{C}$ irradiation becomes important, lowering $\Sigma_{\max }$. The critical density the disc must reach before an outburst is significantly less and the recurrence time between outbursts is decreased. See Sect. 6.1 for details.

have almost the same total mass as they enter quiescence (Fig. 17).

In a truncated disc the drift of material in quiescence will change with $\dot{M}_{\text {tr }}$ because the distance it has to travel is rather short. Therefore, the conditions at the beginning of the outburst depend on the mass-transfer rate. In particular, the ignition radius increases with $\dot{M}_{\mathrm{tr}}$ as could be expected: a larger amount of mass from the outer edge diffuses down the disc and can trigger the outburst at a
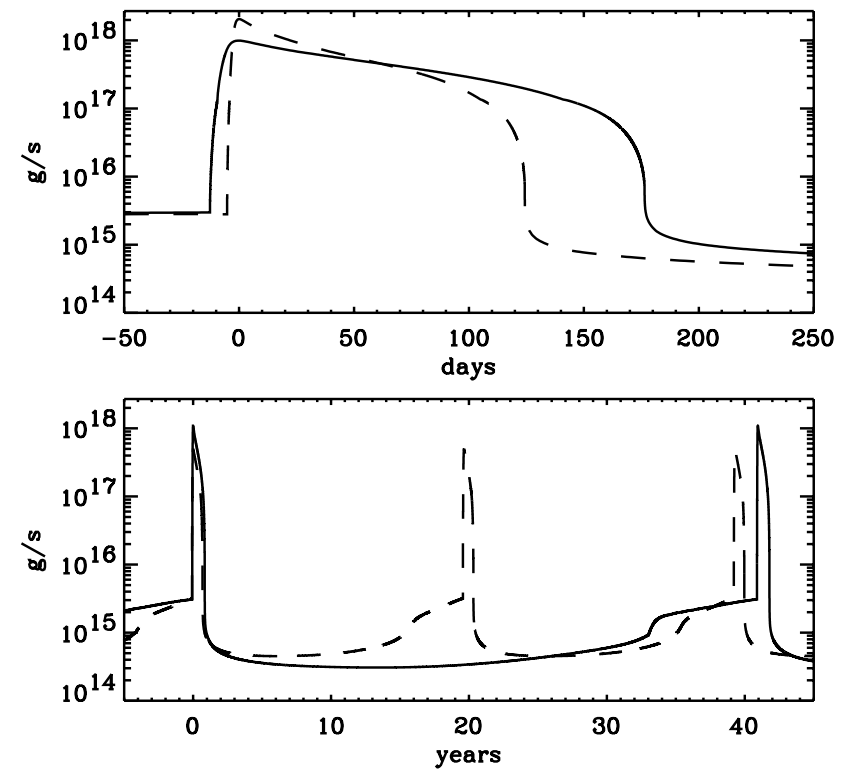

Fig. 16. Effect of changing the viscosity parameters on the outburst time profiles. Bottom: changing $\alpha_{\mathrm{c}}$ modifies the recurrence time (full line $\alpha_{\mathrm{c}}=0.01$, dashed line $\alpha_{\mathrm{c}}=0.02$, both with $\alpha_{\mathrm{h}}=0.1$ ). Top: changing $\alpha_{\mathrm{h}}$ modifies the outburst time evolution (full line $\alpha_{\mathrm{h}}=0.2$, dashed line $\alpha_{\mathrm{h}}=0.4$, both with $\alpha_{\mathrm{c}}=0.02$. The recurrence time is the same $t_{\mathrm{rec}} \approx 20$ years). See Sect. 6.2 for details.

larger radius (but as mentioned earlier outbursts starting at the outer disc edge do not occur). This also increases the total amount of mass in the disc at the onset of the outburst and hence the peak accretion rate (see Sect. 4.1).

For the high mass transfer rates the disc stays fully ionized for a significant fraction of the outburst, leading to a viscous decay. Our model with the highest $\dot{M}_{\text {tr }}$ show this is accompanied by significant variations of the outer radius which produce deviations from the expected exponential (lower panel of Fig. 17).

\subsection{Changing the disc size $R_{\text {out }}$}

The disc size in the model depends on the strength of the tidal truncation term in the angular momentum conservation equation and on the circularization radius. The ratios $R_{\text {circ }} / a$ and $R_{\text {out }} / a$ depend only on the mass ratio $q=M_{2} / M_{1}$. Assuming $q \approx 0.1$, which is reasonable for black hole SXTs, we derive $R_{\text {circ }} / R_{\text {out }} \approx 0.5$ (Papaloizou \& Pringle 1977). In the following, we changed the mean outer disc radius, keeping the above ratio constant. This is equivalent to simulating binaries with the same components but increasing orbital period $P_{\text {orb }} \approx$ $4.6\left(R_{\text {out }} / 10^{11} \mathrm{~cm}\right)^{3 / 2} M_{1}^{-1 / 2}$ hours $(q=0.1)$.

Increasing the disc size makes it more difficult for the heat front to reach the outer disc radius and fully ionize the disc. In small discs the decay is entirely viscous while larger discs show only irradiation-controlled linear decays (Fig. 18). In the case of a viscous exponential decay the 

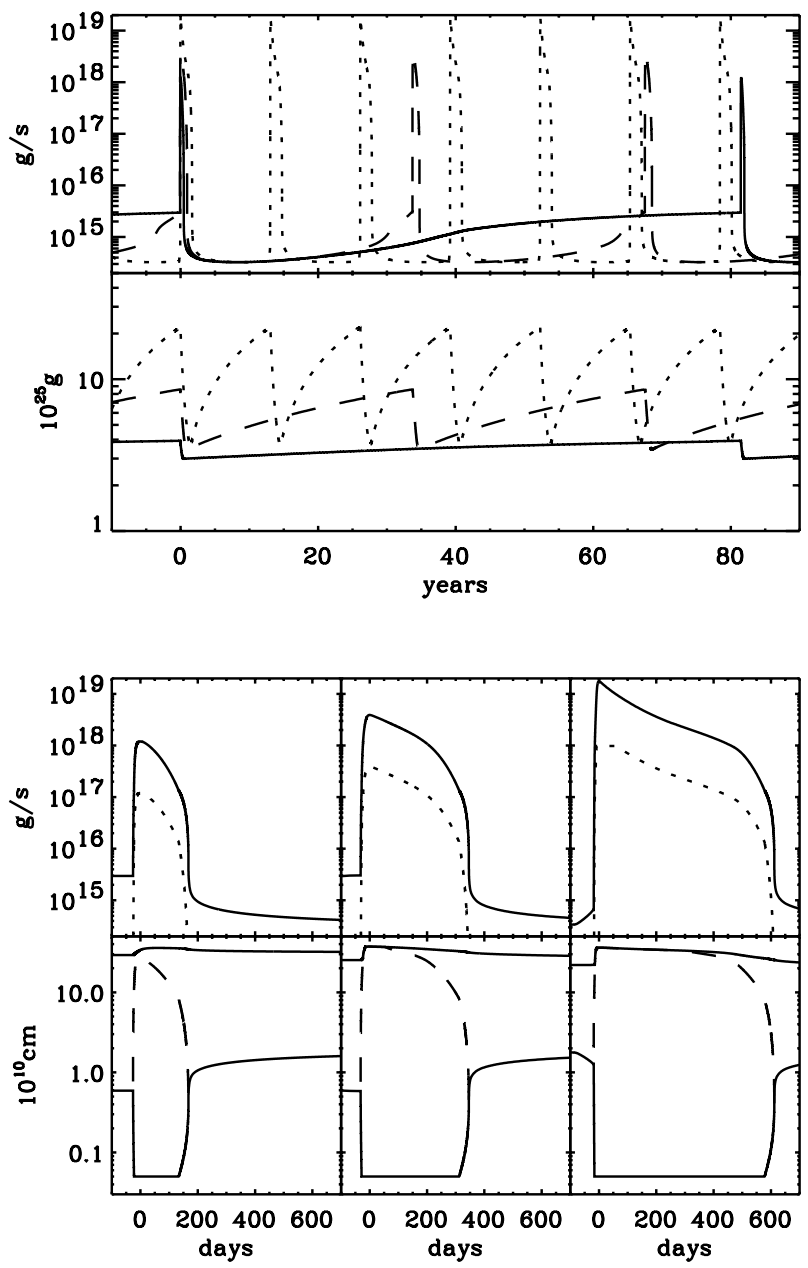

Fig. 17. Effect of changing $\dot{M}_{\mathrm{tr}}$ on the outburst cycles. Top two panels: $\dot{M}_{\text {in }}$ and $M_{\text {disc }}$ for $\dot{M}_{\mathrm{tr}} / 10^{16} \mathrm{~g} \mathrm{~s}^{-1}=, 0.5$ (full line), 5 (dashed line) and 50 (dotted line). The bottom panels zoom on the outburst for the same models, showing $\dot{M}_{\text {in }}$ (full line) and $\dot{M}_{\text {irr }}$ (dotted line), $R_{\text {out }}, R_{\text {in }}$ and the transition radius between the hot and cold regions (dashed line). The other parameters of the models are $M_{1}=7 M_{\odot}, R_{\text {out }} \approx 2.5 \times 10^{11} \mathrm{~cm}$, $\alpha_{\mathrm{h}}=0.2, \alpha_{\mathrm{c}}=0.02$ and $\mathcal{C}=5 \times 10^{-3}$. See Sect. 6.3 for details.

decay rate increases with smaller disc radius in accordance with Eqs. (10)-(11).

The ignition radius of the outburst increases for smaller disc sizes since the diffusion timescale is shorter. Smaller discs are also less massive and, therefore, take less time to replenish than large discs for the same mass transfer rate: the time spent in quiescence is shorter. This also leads to smaller outburst peaks (Sect. 4.1).

\subsection{Changing $M_{1}$}

Finally, we vary the mass of the accreting object $M_{1}$. Irradiation is implicitely assumed to be the same since the prescription for $T_{\text {irr }}$ (Eq. (8)) does not depend on $M_{1}$. $\Sigma_{\max }$ and $\Sigma_{\min }$ have some dependence on $M_{1}$ (Eqs. (3) and (5)) but their influence on the outburst is negligible compared to the changes induced by evaporation. The
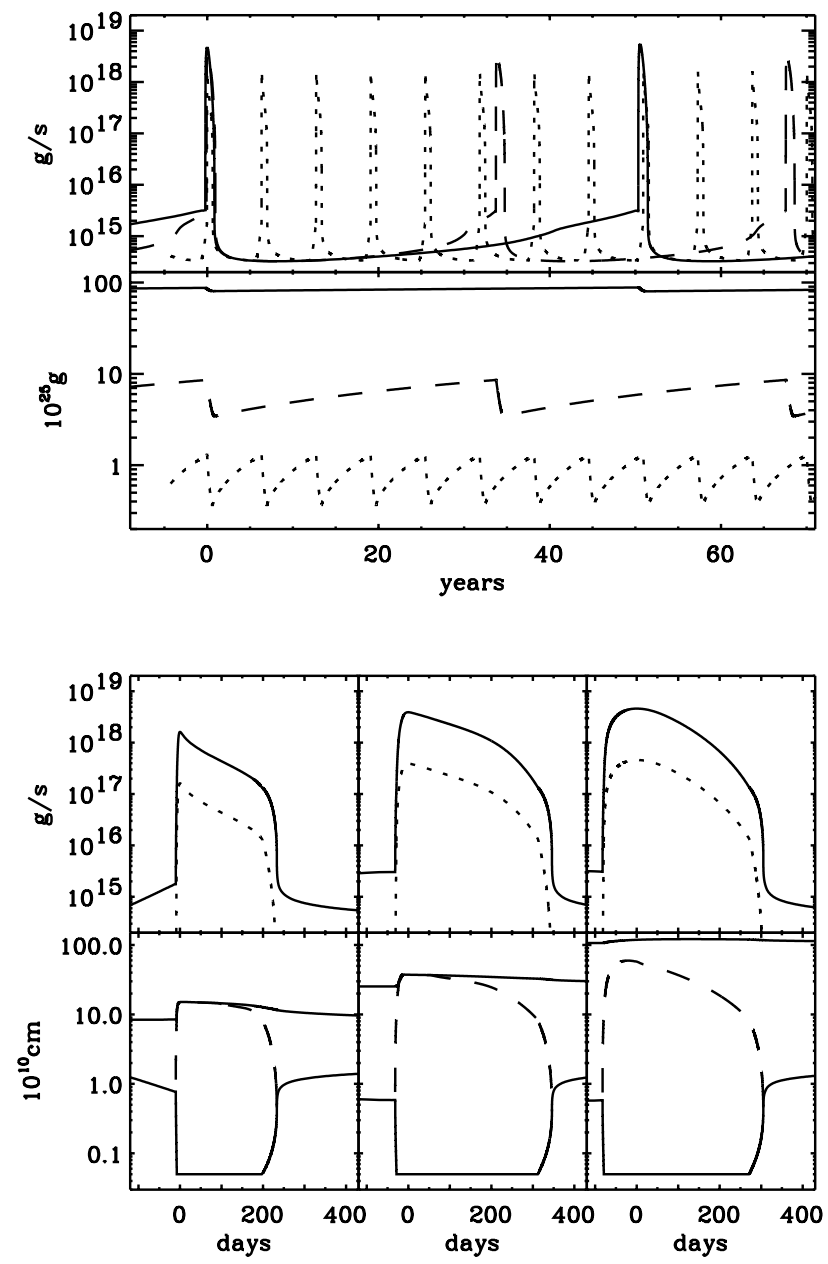

Fig. 18. Effect of changing $R_{\text {out }}$ on the outburst cycles. Top two panels: $\dot{M}_{\text {in }}$ and $M_{\text {disc }}$ for $<R_{\text {out }}>/ 10^{10} \mathrm{~cm}=9.3$ (dotted line), 25.7 (dashed line), 107 (full line). The bottom panels zoom on the outburst for the same models, showing $\dot{M}_{\text {in }}$ (full line) and $\dot{M}_{\text {irr }}$ (dotted line), $R_{\text {out }}, R_{\text {in }}$ and the transition radius between the hot and cold regions (dashed line). The other parameters of the models are $M_{1}=7 M_{\odot}, \dot{M}=5 \times 10^{16} \mathrm{~g} \mathrm{~s}^{-1}$, $\alpha_{\mathrm{h}}=0.2, \alpha_{\mathrm{c}}=0.02$ and $\mathcal{C}=5 \times 10^{-3}$. See Sect. 6.4 for details.

strength of evaporation depends strongly on $M_{1}$ through $\dot{M}_{\text {Edd }}$ and $R_{\mathrm{S}}$ in Eq. (14): $\dot{M}_{\mathrm{ev}} \propto M_{1}^{3}$ (this cannot be seen in Menou et al. 2000 where by error the evaporation formula for neutron-star SXT outbursts uses $\left.M_{1}=6 M_{\odot}\right)$. In outburst, this modifies the accretion rate at which evaporation sets in which in turn reduces the length of the outburst for lower $M_{1}$ (see also Meyer \& Meyer-Hofmeister 2000 who studied extensively the $M_{1}$ dependence of the instability cycle with evaporation, but without irradiation, and reached conclusions similar to those of Menou et al. 1999).

In quiescence, the disc around a lower mass compact object is truncated at smaller radii which reduces the inner accretion rate $\dot{M}_{\text {in }}$. Mass is accumulated more easily (Eq. (17)), leading to shorter recurrence time (Fig. 19). The lower accretion rates in the evaporated disc in quiescence do not necessarily translate into lower quiescent luminosities. In our assumptions, the increase in accretion 


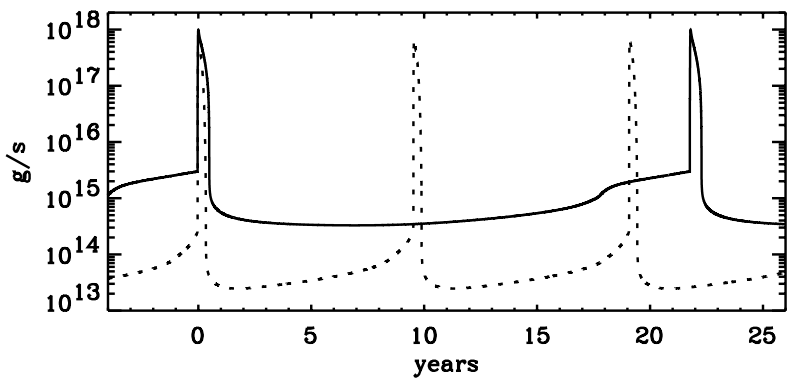

Fig. 19. Effect of changing $M_{1}$ on the outburst cycles of the inner mass accretion rate $\dot{M}_{\text {in }}$. The model of Sect. 5 is shown by the full line $\left(M_{1}=7 M_{\odot}\right)$. The same model but with $M_{1}=$ 1.4 $M_{\odot}$ is shown by the dotted line. See Sect. 6.5 for details.

efficiency implied by a lower $R_{\text {in }}\left(\epsilon \propto R_{\text {in }}^{-2}\right)$ compensates for the lower $\dot{M}_{\text {in }}$. Plotting $\dot{M}_{\text {irr }}=\epsilon \dot{M}_{\text {in }}$ instead of only $\dot{M}_{\text {in }}$ as in Fig. 19, we find $\dot{M}_{\text {irr }} \approx 10^{10-11} \mathrm{~g} \mathrm{~s}^{-1}$ for both $M_{1}=1.4 M_{\odot}$ and $7 M_{\odot}$. The actual efficiency of a $1.4 M_{\odot}$ neutron star may be much higher in quiescence than what we assume here. It might actually be more correct to assume $\epsilon$ is always 0.1 for the neutron star since the accreted matter will radiate at the surface. In this case, the luminosity in quiescence deduced from $\dot{M}_{\text {in }}$ will be higher than for a black hole primary. However, a propeller effect might significantly reduce the actual mass accretion rate onto a neutron star in quiescence (Menou et al. 1999c) making the distinction between black holes and neutron stars less obvious.

\section{Discussion}

Our most basic hypothesis is that the outbursts of SXTs are due to the thermal-viscous instability in a thin accretion disc. There is little doubt that LMXBs do possess thin accretion discs, at least far from the compact object, while the wide applicability of the thermal-viscous instability in CVs gives strong support to its existence in LMXBs. It has long been known that models of dwarf novae require a lower viscosity in quiescence than in outburst. Models with $\alpha_{\mathrm{c}}=\alpha_{\mathrm{h}}$ predict low-amplitude outbursts with short cycles which do not agree with observations (Smak 1984; Lin et al. 1985). The upper hot branch of the local thermal equilibrium curve ( $S$-curve) is characterized by a high viscosity while the opposite is true for the lower cold branch. Our "standard" model refers to the thermal-viscous instability in such a two- $\alpha$ thin disc.

These two- $\alpha$ models, although not without problems (Smak 2000; Lasota 2001), have basic properties (recurrence time, amplitude and length of some type of outbursts) which compare reasonably well with observations, at least for some classes of CVs (for instance, the U Gem type dwarf novae). The standard model as applied to CVs offers a background upon which more detailed scenarios have been constructed (with various success) to explain observational features such as the UV delay, superoutbursts, superhumps, standstills etc. (see Lasota 2001 for a review). None of these features have (up to now) threatened the basic premise that the outbursts are triggered by the thermal-viscous instability in a two- $\alpha$ thin disc.

In contrast, the standard DIM was never able to reproduce even the most basic features of any SXT. In particular, the impossibility of obtaining anything like the observed exponential decays casted serious doubts on the validity of using the two- $\alpha$ assumption to represent the viscosity in these discs, prompting the use of more complex formulations (Cannizzo et al. 1995). After all, disc models with ab initio treatment of the viscosity are still some way off and the $\alpha$-disc could be an oversimplification. However, one must ensure that the failure of models to reproduce observations are really due to the viscosity prescription, and not to the fact that some physical effects have not been correctly taken into account.

Indeed, in a number of cases it has been shown that the disagreements between models and observations resulted not so much from the assumptions on the viscosity but from the physics which was or was not included. In $\mathrm{CVs}$, studies that successively included a variable disc size, evaporation of the central regions, irradiation by the white dwarf, stream-impact and tidal dissipation heating have gradually led to increased improvement in the agreement between observations and detailed predictions of models (the most recent being Hameury et al. 1998, 1999, 2000; Buat-Ménard et al. 2001). For example, it was shown by Smak (1998) that the failure of models to reproduce the observed UV delay largely resulted from the use of a fixed outer boundary condition and too small discs, making outside-in outbursts quite difficult to obtain. And of course, it was early realized that parameters such as the mass transfer rate from the secondary had to vary in order to account for the standstills of the Z Cam stars (Meyer \& Meyer-Hofmeister 1983), not to mention the necessity of using a number of grid points large enough to resolve fronts (Cannizzo 1993). It will therefore come as no surprise that the failure of the two- $\alpha$ model in SXTs was more indicative of missing physics than inadequate assumptions on the viscosity.

In the following we review the results of the previous sections to show how the fundamental properties of evaporation and irradiation lead to better agreement with observations within the two- $\alpha$ model. We then critically examine possible failures of this model in light of detailed comparisons with observations.

\subsection{Fundamental properties of the model}

It has long been known that the standard disc instability model fails on three fundamental accounts: (a) the timescales of the cycles are wrong with short outbursts and recurrence times; (b) the decay from outburst is dominated by reflare episodes which are not observed; (c) even the full conversion to X-rays of the rest mass energy of the accreted material could not power the observed luminosities if the quiescent disc reaches down to the 
compact object. All of these are unavoidable consequences of the model which do not depend on the detailed assumptions. In the standard DIM thermal cooling starts as soon as the outburst peak is reached. This proceeds on a short timescale since an unstable ring evolves on a thermal timescale. This results in (a). Problem (b) is the consequence of the high densities behind the cooling front and of the decreasing critical density needed to trigger an outburst as the cooling front propagates inwards. Unless the disc can be emptied in outburst, which is difficult because of (a), reflares are unavoidable. Problem (c) is also due to the decreasing critical density: a quiescent disc must have densities below the critical $\Sigma_{\max }$ which implies extremely low accretion rates if the disc extends to small radii. Either the quiescent $\mathrm{X}$-rays are not produced by accretion (which is possible for neutron stars: Rutledge et al. 2000; but very unlikely for black-holes: Lasota 2000) or the disc is truncated so that the mass flow through the inner region (assuming it is constant) is high. This was first suggested in the context of dwarf novae to explain the optical to EUV delay (Meyer \& Meyer-Hofmeister 1994).

This is a particularly attractive scenario when the inner region is a hot ADAF flow (Lasota et al. 1996). An ADAF of varying size and accretion rate produces harder or softer spectra which compare well with the observed X-ray spectral states of SXTs. With this in mind, Menou et al. (2000) included disc truncation in quiescence to the standard DIM. Yet, their models required values of $\alpha$ lower than those usually assumed in dwarf novae and were not able to reproduce the short rise times observed in SXTs. In principle one would hope the viscosity behaves in the same way between CVs and $\mathrm{LMXBs}^{3}$. Another way to get long recurrence times would be to have the rate of evaporation of the disc in quiescence almost match the mass transfer rate from the secondary (Meyer \& Meyer-Hofmeister 1999). Again this is not satisfactory: this requires finetuning of the evaporation process and/or strongly implies that most of the LMXB population would be cold and stable (Menou et al. 1999b; Meyer \& Meyer-Hofmeister 2000) with the outbursts triggered by some unspecified mechanism (e.g. increased mass transfer from the secondary). This is not unlikely, but is disappointing in that most SXTs would then be unusual in some respect with little prospect for unifying explanations.

Irradiation provides an adequate resolution of the three problems mentioned without having to assume a very low quiescent viscosity. Irradiation is an observational fact and simple estimates show its impact on the outburst cycles must be important. Irradiation is also the only major difference between CVs and LMXBs. In CVs the irradiation flux is at lower energies and originates from a (relatively) large white dwarf. In LMXBs the flux is at high energies and (presumably) originates from the central regions of the disc or from a corona. The distribution

\footnotetext{
${ }^{3}$ But one should keep in mind that there is a subclass of dwarf novae (the WZ Sge systems) for which low values of the viscosity in quiescence have been invoked.
}

and amplitude of the fluxes are totally different. As such it was a prime candidate for the most important missing piece of physics in SXT models.

In Sect. 4 we have seen how irradiation drastically changes the outburst. The key point is that the thermal instability is suppressed when $T_{\text {irr }} \gtrsim 10^{4} \mathrm{~K}$. In this sense, the results shown in this paper depend little on the assumptions made on the irradiation flux. A disc will show slow exponential decays for as long as a large enough irradiating flux can keep the outer disc above hydrogen ionization and the exact formulation of $F_{\text {irr }}$ is unimportant. The first major consequence is therefore to prolong the outburst by providing a significant additional source of heating.

During the thermal decay, the irradiation flux determines the location of the cooling front. In general the cooling front can only appear after most of the mass of the disc has already been accreted. Even if irradiation stops too quickly and the outburst ends prematurely, the densities in the disc will still be much lower than when irradiation is not included. This is our second major consequence since this makes reflares impossible and also lengthens the quiescence time needed to rebuild the disc mass.

In our models we have neglected the possible impact of the X-ray spectrum on irradiation heating. We have implicitly assumed it was soft enough to confine heating to a small layer at the top of the disc (Lyutyi \& Sunyaev 1976). On the other hand, hard X-rays should penetrate deeper and deposit energy all along the vertical layer. This should not significantly change the main conclusions of this paper for a simple reason: whatever the distribution of irradiation heating in the layer it will still globally heat the ring and displace its thermal equilibrium $S$-curve to lower $\Sigma$. This can be seen in Figs. 1, 2 of El-Khoury \& Wickramasinghe (1999). Even hard X-rays will prolong the outburst until sufficiently low densities can be reached for the disc to cool.

In an irradiated disc extending to low radii problem (c) remains. By including truncation in Sect. 5, we have shown that very long recurrence times are easily obtained. These result naturally from irradiation which forces the disc to accrete more during outburst and from evaporation which requires the disc to reach higher densities before an outburst can be triggered again. There is no need for very low values of $\alpha$ in quiescence to reach recurrence timescales of tens of years.

An adequate model of SXTs can therefore be obtained by including some form of irradiation in outburst and some form of evaporation in quiescence. Regardless of the detailed assumptions this will necessarily lead to long outbursts with slow decays and long quiescence times for values of the viscosity which are similar to those used in models of CVs.

\subsection{Comparison with observations}

The observed timescales for the rise (1-14 days), decay (8-40 days), and total outburst duration (40-300 days) 


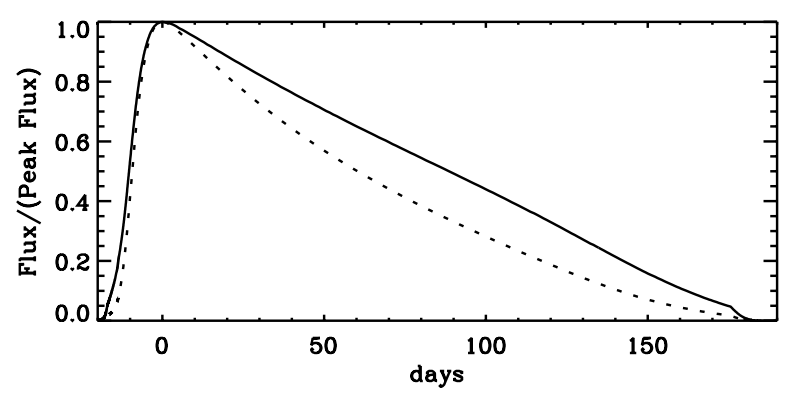

Fig. 20. $V$-band optical flux (full line) compared to $\dot{M}_{\text {irr }}=$ $\epsilon \dot{M}_{\text {in }}$ (dotted line) during an outburst of the model of Sect. 5 (Fig. 10). $\dot{M}_{\text {irr }}$ is taken as an indicator of the X-ray flux. Both are normalized to their peak value. The optical flux is halved in $\approx 100$ days while the $\mathrm{X}$-ray flux is halved in $\approx 60$ days.

defined by Chen et al. (1997) are well reproduced by our models in very general conditions i.e. with no fine tuning of any parameter, including $\alpha$. The model also predicts recurrence timescales anywhere between a year to tens of years, as observed. The amplitudes of the outbursts are high with peaks at $10^{37-38} \mathrm{erg} \mathrm{s}^{-1}$ (assuming $L_{x}=$ $0.1 \dot{M}_{\text {peak }} c^{2}$ ), all consistent with observations. Weak outbursts (i.e. with less irradiation) have shorter durations than strong ones just as noticed by Chen et al. (1997).

Our models, taken at face value, do not make accurate predictions for the observed outburst amplitudes in neutron stars and black holes because the efficiency $\epsilon$ of the ADAF in quiescence is not properly calculated here. It would be particularly interesting to see whether quiescent discs with neutron star primaries have similar luminosities as black hole systems as briefly suggested in Sect. 6.5. In this case, this would support the idea that the higher quiescent luminosities of NS SXTs is due to thermal emission from the surface of the NS heated by accretion during the outburst and hence proves indirectly the existence of horizons in BHs (Narayan et al. 1997; Menou et al. 1999c)

A clear feature of the model which is observed in SXTs is the slower optical decay time. This is due to irradiation which, under very general conditions, can keep the cold outer disc hotter than usual during the propagation of the cooling front (Fig. 20). Figure 12 shows the disc behind the front is dominated by irradiation and almost isothermal. Obviously, the peak optical flux is much higher when the disc is irradiated than when it is not.

There is observational evidence pointing towards a delay between the outburst rise in the optical and the rise in the X-rays. This has been most evident in GRO J1655-40 where a 6 day delay was observed (Orosz et al. 1997). As stressed by Hameury et al. 1997, this delay and the lightcurve shape cannot be explained by an outside-in outburst in a disc extending down to the last stable orbit. The delay is rather the time for the thin disc to replace the hot flow in the inner regions. This happens on a much longer viscous timescale than the timescales on which fronts propagate through the disc. In the early rise, one would expect the optical to rise first due to the propagation of the heat front and the X-rays to form later when the thin disc reaches regions close to the compact object. Whether the outburst starts with an inside-out or an outside-in front matters little.

In the present calculations, the time taken by the thin disc to reach its minimum radius is of the order of a few days (see Sect. 5.1). Although it would be desirable, we cannot provide better tests of the delays as this would require a detailed model of $\mathrm{X}$-ray formation and of irradiation during the early rise (which can drastically change the optical lightcurve). This can be done if one couples an ADAF model with the thin disc and consistently calculates the evolution of both, specifically the thin disc irradiation from the ADAF.

To zero order, the model thus satisfies all major observed properties. Inversely, what can be inferred from an observed lightcurve on the accretion disc? An easily obtained measure is the peak flux of the outburst. The study of Sect. 6 shows this increases with mass accretion rate and disc size (orbital period). The peak luminosity for neutron star primaries is generally smaller than for a black hole with a similar accretion disc. However, this might be dependent on the assumed evaporation law (here evaporation is weaker for a NS). Another parameter of interest, when available, is the recurrence time between outbursts. The models predicts the lowest times for systems closest to the stability limit i.e. with high mass transfer rates for their sizes (or vice-versa). Systems with long orbital periods are more likely to show outbursts deviating from an exponential since the heat front may not reach the outer edge. The strength of irradiation is also important: systems showing little evidence for it would have short outbursts. The above assumes identical values of the viscosities. In practice, the effects of the different parameters are too entangled to reach strong conclusions from an observed lightcurve except, perhaps, in the most extreme cases. Consequently, the prospects of further constraining viscosity from the observed outburst lightcurves are dim. Dwarf novae, if only because of sheer numbers, are a better tool to achieve this goal (e.g. Smak 1999). X-ray observations of SXTs in quiescence will also be very helpful in constraining both the evaporation processes and the viscosity at low temperatures, since very low quiescent viscosities should result in very small X-ray luminosities.

\subsection{Outstanding problems}

A quick review of observed X-ray lightcurves will reveal that these are much more complex than the nicely behaved decays we predict. Indeed, the past few years have seen a number of SXTs detected with lightcurves which seem destined to ruin a model originally based on the canonical A0620-00 (e.g. GRO J1655-40, XTE J1550-564, XTE J1118+480...). These systems typically show consecutive outbursts separated by a few months with the second outburst more akin to a plateau than an exponential. Both the plateau and short quiescence time (if the system returns to quiescence) cannot be explained by the model 
presented here without additional assumptions. Possibilities include increased mass transfer from the secondary after the star was irradiated during the first outburst. This can easily lead to plateau-like lightcurves when mass transfer temporarily stabilizes the disc. Further complications can arise if the disc is large enough that it becomes warped in outburst and, for instance, shadows the secondary (Esin et al. 2000a; Ogilvie \& Dubus 2001). Winds created by strong irradiation can cause significant mass loss resulting in quicker exponential decays (Mineshige et al. 1993).

The most decently behaved SXTs also have some intriguing features. For instance, A0620-00 and GRS 112468 show glitches (in the terminology of Chen et al. 1997) during a decay which otherwise could very well be fitted by the model (see the discussion by Cannizzo 1998b). Our models clearly show these cannot be due to the transition between the viscous and thermal decays as speculated by King \& Ritter (1998). Esin et al. (2000b) proposed that the glitches could be due to increased irradiation in a warped disc while Cannizzo (2000) argued for evaporation in the outer disc. Both these possibilities have been explored and will be dealt with in a future paper. Even more puzzling are the multiple "mini-outbursts" observed at the end of the outburst of e.g. GRO J0422+32. These bear many similarities to some outbursts of WZ Sge type dwarf novae (e.g. Kuulkers 2000), suggesting a common mechanism. The mini-outbursts are in no way comparable to the reflares discussed above. There are no satisfying explanations for the mini-outbursts.

\section{Conclusion}

We have shown that, under general assumptions, a model of the thermal viscous instability including both the effects of irradiation and evaporation of the inner disc in quiescence led to outburst cycles with timescales, amplitudes and slow decays which compare well with observations. This does not require any fine-tuning or modifications of the standard viscosity assumptions used in models of CVs. Even very moderate amounts of irradiation significantly increase the length of the outburst, its shape and the recurrence timescale.

These results depend little on the detailed choices made in describing either the irradiation flux or the evaporation law. The assumptions are most important during the rise and at the end of the outburst. Further comparisons with observations will require these to be known more precisely. The next logical step will be to consistently include an ADAF model in the evaporated region so as to be able to calculate at each moment the X-ray spectrum and flux seen by the disc (and the observer).

The wide applicability of the DIM in CVs and SXTs is therefore confirmed. The importance of a careful assessment of the necessary physics cannot be more stressed than for the case of SXTs whose basic features can only be reproduced by the model when the effects of irradiation and evaporation are included.
Yet, the lightcurves of SXTs show more complexity than the simple exponential or slow decays we predict. This is suggestive of still more missing physics, possibly variations in the mass transfer rate from the secondary or complex behaviour of the irradiation flux. These are difficult to model and most certainly vary from object to object. In this sense, more detailed modelling might not prove very rewarding. Also the prospects of using SXTs to constrain viscosity beyond the simple two- $\alpha$ model are poor. The finer effects of viscosity are hidden amongst the complex interactions, between other physical effects. However, the dynamical models show viscosity must behave in the same way in CVs and SXTs which, at least, supports a common origin.

Acknowledgements. We thank Hans Ritter for his very detailed report. GD is supported by the TMR network "Accretion on to Black Holes, Compact Stars and Protostars" (contract number ERBFMRX-CT98-0195). This work was also supported by a Spinoza award to E.P.J. van den Heuvel and by the Programme National de Physique Stellaire of the CNRS.

\section{References}

Buat-Ménard, V., Hameury, J.-M., \& Lasota, J.-P. 2001, A\&A, 366,612

Begelman, M. C., McKee, C. F., \& Shields, G. A. 1983, ApJ, 271, 70

Brown, E. F., Bildsten, L., \& Rutledge, R. E. 1998, ApJ, 504, 195

Cannizzo, J. K. 1993, ApJ, 419, 318

Cannizzo, J. K. 1998a, Wild Stars in the Old West: Proceedings of the 13th North American Workshop on Cataclysmic Variables and Related Objects, ed. S. Howell, E. Kuulkers, \& C. Woodward, San Francisco, ASP, 308

Cannizzo, J. K. 1998b, ApJ, 494, 366

Cannizzo, J. K. 2000, ApJ, 534, L35

Cannizzo, J. K., \& Wheeler, J. C. 1984, ApSS, 55, 367

Cannizzo, J. K., Ghosh, P., \& Wheeler, J. C. 1982, ApJ, 260, $\mathrm{L} 83$

Cannizzo, J. K., Lee, H. M., \& Goodman, J. 1990, ApJ, 351, 38

Cannizzo, J. K., Chen, W., \& Livio, M. 1995, ApJ, 454, 880

Chen, W., Shrader, C. R., \& Livio, M. 1997, ApJ, 491, 312

Czerny, B., Czerny, M., \& Grindlay, J. E. 1986, ApJ, 311, 241

Dubus, G. 2000, in Proceedings of the 19th Texas Symposium held in Paris, Nucl. Phys. B, Proc. Suppl. 80, CD-ROM

Dubus, G., Lasota, J.-P., Hameury, J.-M., \& Charles, P. A. 1999, MNRAS, 303, 139

El-Khoury, W., \& Wickramasinghe, D. 1999, MNRAS, 303, 380

Esin, A. A., McClintock, J. E., \& Narayan, R. 1997, ApJ, 489, 865

Esin, A. A., Lasota, J.-P., \& Hynes, R. 2000a, A\&A, 354, 987

Esin, A. A., Kuulkers, E., McClintock, J. E., \& Narayan, R. 2000b, ApJ, 532, 1069

Gammie, C. F., \& Menou, K. 1998, ApJ, 482, L75

Hameury, J.-M., Lasota, J.-P., McClintock, J. E., \& Narayan, R. 1997, ApJ, 489, 234

Hameury, J.-M., Menou, K., Dubus, G., Lasota, J.-P., \& Huré, J.-M. 1998, MNRAS, 298, 1048 
Hameury, J.-M., Lasota, J.-P., \& Dubus, G. 1999, MNRAS, 303,39

Hameury, J.-M., Lasota, J.-P., \& Warner, B. 2000, A\&A, 353, 244

Hoshi, R. 1984, PASJ, 36, 785

Ichikawa, S., \& Osaki, Y. 1994, in Theory of Accretion Disks-2, ed. W. Duschl et al. (Kluwer Academic Pub., Dordrecht), 169

Idan, I., \& Shaviv, G. 1996, MNRAS, 281, 615

Kim, S.-W., Wheeler, J. C., \& Mineshige, S. 1999, PASJ, 51, 393

King, A. R. 1998, MNRAS, 296, L45

King, A. R., \& Ritter, H. 1998, MNRAS, 293, L42

de Kool, M., \& Wickramasinghe, D. 1999, MNRAS, 307, 449

Kuulkers, E. 2000, NewAR, 44, 27

Lasota, J.-P. 1996, in Cataclysmic Variables and Related Objects, IAU Symp. 158, ed. A. Evans, \& J. H. Wood (Kluwer, Dordrecht), 385

Lasota, J.-P. 1999, Phys. Rep., 311, 95

Lasota, J.-P. 2000, A\&A, 360, 575

Lasota, J.-P. 2001, New Astron. Rev., 45/7, 449

Lasota, J.-P., Narayan, R., \& Yi, I. 1996, A\&A, 314, 813

Lin, D. N. C., Papaloizou, J., \& Faulkner, J. 1985, MNRAS, 212,105

Lyubarskii, Y. E., \& Shakura, N. I. 1987, SvAL, 13, 386

Lyutyi, V. M., \& Sunyaev, R. A. 1976, SvA, 20, 290

Menou, K., Hameury, J.-M., \& Stehle, R. 1999a, MNRAS, 305, 79

Menou, K., Narayan, R., \& Lasota, J.-P. 1999b, ApJ, 513, 811

Menou, K., Esin, A. A., Narayan, R., et al. 1999c, ApJ, 520, 276

Menou, K., Hameury, J.-M., Lasota, J.-P., \& Narayan, R. 2000, MNRAS, 314, 498

Meyer, F. 1984, A\&A, 131, 303

Meyer, F. 1986, MNRAS, 218, 7

Meyer, F., \& Meyer-Hofmeister, E. 1982, A\&A, 106, 34

Meyer, F., \& Meyer-Hofmeister, E. 1983, A\&A, 121, 29

Meyer, F., \& Meyer-Hofmeister, E. 1994, A\&A, 288, 182

Meyer, F., \& Meyer-Hofmeister, E. 1999, A\&A, 348, 154
Meyer, F., \& Meyer-Hofmeister, E. 2000, A\&A, 355, 1073

Meyer, F., Liu, B. F., \& Meyer-Hofmeister, E. 2000, A\&A, 361, 175

Mineshige, S., \& Kusunose, M. 1993, PASJ, 45, 113

Mineshige, S., \& Wheeler, J. C. 1989, ApJ, 343, 241

Mineshige, S., Tuchman, Y., \& Wheeler, J. C. 1990, ApJ, 359, 176

Mineshige, S., Yamazaki, T., \& Ishizaka, C. 1993, PASJ, 45, 707

Narayan, R., Mahadevan, R., \& Quataert, E. 1999, in Theory of Black Hole Accretion Discs, ed. M. A. Abramowicz et al. (Cambridge University Press), 148

Narayan, R., McClintock, J. E., \& Yi, I. 1996, ApJ, 457, 821

Narayan, R., Garcia, M. R., \& McClintock, J. E. 1997, ApJ, 478, L79

Ogilvie, G. I, \& Dubus, G. 2001, MNRAS, 320, 485

Orosz, J. A., Remillard, R. A., Bailyn, C. D., \& McClintock, J. E. 1997, ApJ, 478, L83

Osaki, Y. 1995, PASJ, 47, 47

Osaki, Y. 1996, PASP, 108, 39

Papaloizou, J., \& Pringle, J. E. 1977, MNRAS, 181, 441

Papaloizou, J., \& Pringle, J. E. 1985, MNRAS, 217, 387

van Paradijs, J. 1996, ApJ, 464, L139

van Paradijs, J., \& McClintock 1994, A\&A, 290, 133

Rutledge, R. E., Bildsten, L., Brown, E. F., Pavlov, G. G., \& Zavlin, V. E. 2000, ApJ, 529, 985

Shaviv, G., Wickramasinghe, D., \& Wehrse, R. 1999, A\&A, 344,639

Shakura, N. I., \& Sunyaev, R. A. 1973, A\&A, 24, 337

Smak, J. 1984, Acta Astron., 34, 161

Smak, J. 1993, Acta Astron., 43, 101

Smak, J. 1998, Acta Astron., 48, 677

Smak, J. 1999, Acta Astron., 49391

Smak, J. 2000, NewAR, 44, 171

Tanaka, Y., \& Shibazaki, N. 1996, ARA\&A, 34, 607

Tuchman, Y., Mineshige, S., \& Wheeler, J. C. 1990, ApJ, 359, 164

Vishniac, E. T. 1997, ApJ, 482, 414

Vishniac, E. T., \& Wheeler, J. C. 1996, ApJ, 471, 921 